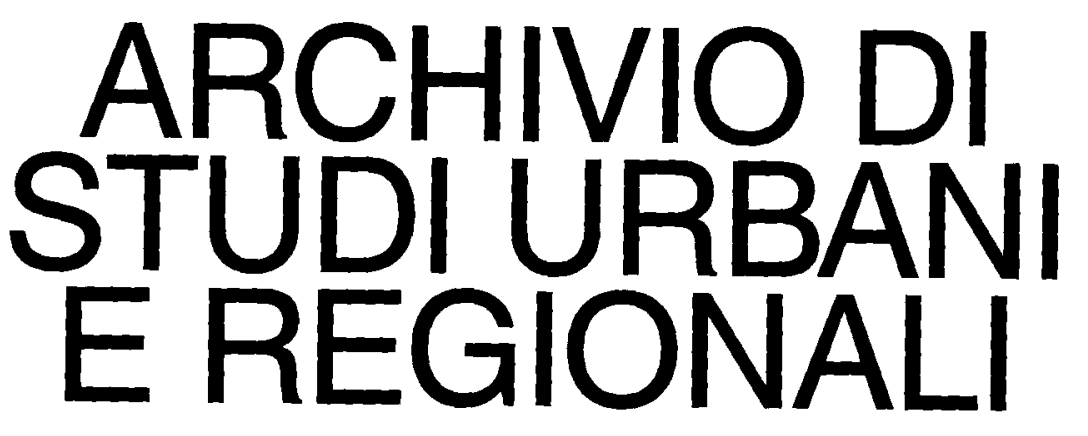

FrancoAngeli - anno XXXIX - n. 93, 2008

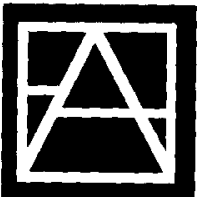


Franceseo Musco, Cambiamenti climatici, politich di adattamento e mitigazione: una prospettiva urhana

Loris Serafino e Sandro Fabbro, «Chi non innova è perdento's. Un'analisi critica della visione strategica di regione in Friuli Venezia Giulia

Alessandro Crociata e Paolo Veneri, II seftore culturale nello sviluppo urhano: il caso di Ancona

Elena Lorenzetto, Uno squardo semiotico sul Territorio. Presentazione di un lavoro di analisi della cittá diffusa del Veneto cintrale

\section{Dibattito}

Orientamenti: Milano, Roma, Bari e Palermo, a cura di Francesco Indovina

Elena Granata, Milano e la sohria inquictudine di una metropoli in cerca di fituro

Laura Valeria Ferretti. Quculche riffessione sulle politiche urbane a Roma

Nicola Martinelli e Maria Raffaella Lamacchia, Buri: due governi urbani. Elementi di persistenza é discontimutita

Teresa Cannarozzo, Palermo: cronache del terzo millennio

Recensioni

Gli autori di questo numero 


\title{
PALERMO: CRONACHE DEL TERZO MILLENNIO
}

\author{
di Teresa Cannarozzo
}

\section{Il quadro politico}

Come'è noto, le elezioni nazionali e regionali del 13 e 14 aprile 2008 hanno consegnato l'Italia e la Sicilia al centro-destra cancellando del tutto la rappresentanza politica delle forze politiche a sinistra del neonato Partito Democratico. Le ragioni della disfatta sono molteplici e ancora da chiarire; c'è però un elemento di novità che va sottolineato e cioè l'affermazione anche a livello nazionale di un nuovo movimento politico, in pista da pochi anni, apparentato con la Lega di Bossi e con il centro-destra: il Movimento politico per l'autonomia (Mpa) del siciliano Raffaele Lombardo, deputato europeo, eletto trionfalmente presidente della Regione con il $65 \%$ dei consensi. Lo schieramento di centro-sinistra che aveva candidato l'autorevole senatrice del Pd Anna Finocchiaro non è andato al di là di un misero $30 \%$, con un risultato di dieci punti al di sotto di quello conseguito da Rita Borsellino nella sfida a Salvatore Cuffaro in occasione delle regionali del $2006^{\prime}$. ¿ E interessante notare che in Sicilia la Sinistra Arcobaleno, guidata da Rita Borsellino, ha mancato per un soffio il superamento dello sbarramento del $5 \%$ previsto dalla legge elettorale regionale, a differenza che nel resto d'Italia dove si è attestata sul 3\%. Questi risultati nazionali e la pessima performance siciliana del Partito democratico sono un tema inquietante che andrebbe analizzato con coraggio e rigore: cosa che sicuramente non avverrà.

$\grave{E}$ il caso di ricordare che in Sicilia si è votato anticipatamente perché il presidente Cuffaro è stato costretto a dimettersi, suo malgrado, nel gennaio 2008 , per una condanna in primo grado a cinque anni e l'interdizione perpetua dai pubblici uffici, per favoreggiamento aggravato a soggetti rivelatisi mafiosi e rivelazione di segreti d'ufficio. Le dimissioni di Cuffaro hanno provocato per la prima volta nella storia lo scioglimento dell'Assemblea regionale e ciò dà la misura della decadenza verticale delle istituzioni e del-

1. Nelle elezioni regionali del 2006, il centro-sinistra aveva schicrato Rita Borsellino, vice presidente di Libera e sorella del giudice Paolo Borsellino, uceiso dalla mafia nelle stragi del 1992. Aveva vinto Cuffaro ma con una differenza di soli 200.000 voti. 
la politica siciliana. Le foto di Cuffaro con un vassoio di cannoli in mano che festeggiava la "leggerezza" della condanna hanno fatto il giro del mondo e ci hanno fatto vergognare di essere siciliani, ma questo non ha impedito all'Udc di Casini di candidare Cuffaro (vice segretario nazionale del partito) come capolista al Senato. La scelta, assai opinabile dal punto di vista etico, ha pagato dal punto di vista politico perché gli unici senatori che l'Udc ha piazzato sono solo tre siciliani.

II cuffarismo, che è stato codificato come pratica politica e sociale, con contenuti specifici, produce fedeltà, affezione e uno straordinario consenso elettorale, a cui i vertici nazionali dell'Udc non hanno inteso rinunciare, invocando un garantismo di convenienza, basato sull'ipotetica modificabilità della sentenza di primo grado. D'altronde, nulla di nuovo sotto il sole: anche Andreotti e la Democrazia cristiana. negli anni 70 , non facevano gli schizzinosi con i voti portati dai luogotenenti siciliani Salvo Lima e Vito Ciancimino (Cannarozzo, 2000).

I sociologi e i politologi dovranno però attrezzarsi a studiare anche il lombardismo perché i risultati conseguiti da Raffacle Lombardo, con il suo Mpa, sono ancora superiori $i^{2}$. C'è da dire che $\mathrm{i}$ due hanno molto in comune: origini politiche all'interno della $\mathrm{Dc}$, apprendistato alla corte di Calogero Mannino, autorevole politico democristiano dell'agrigentino, più volte ministro e con qualche pendenza giudiziaria non del tutto risolta. Hanno in comune una laurea in medicina e una straordinaria capacità di mobilitazione di ampi segmenti della società che forse va letta e analizzata al di là del paradigma troppo semplicistico del voto di scambio o del voto mafioso. Per la verità, durante la campagna elettorale non è mancato un appello indiretto agli elettori mafiosi; cosi è stata interpretata da molti la dichiarazione del senatore palermitano Marcello Dell'Utri, del PdL, sull'eroismo manifestato dal famoso stalliere di Arcore, il mafioso siciliano, Vittorio Mangano, a servizio di Berlusconi, nel non avere mai fatto dichiarazioni ai pubblici ministeri che avrebbero potuto compromettere gli amici comuni.

In attesa di rigorose ed improbabili analisi del voto siciliano, ivi compresi l'astensionismo e i voti andati alla lista degli amici di Beppe Grillo, il grande successo elettorale di Lombardo può anche essere spiegato con l'offerta di candidature a molti esponenti di successo della cosiddetta società civile: professionisti, imprenditori, commercianti.

Lombardo ha riproposto con forza il tema della malaugurata autonomia regionale, la costruzione del Ponte sullo Stretto, agevolazioni fiscali alle imprese che investono in Sicilia, un megacentro direzionale dove concentrare tutti gli uffici della Regione e la necessità di acchiappare tutti i fondi europei che saranno erogati per l'ultima volta nell'arco temporale 20072013. Argomenti vecchi, su cui molto si è detto e si è scritto.

2. Si veda l'articolo di M. Centorrino, «La "pancia" della Sicilia», La Repubblica-Palermo. 3 mag. 2008.
Il nuovo $\operatorname{Pr}$ perché ha prete togliendo poltr moltiplicate le rie, auto di ser

Nel panorar contestazioni o inefficienza de nazionale nei dell'Assessore sua determinaz più importanti UNESCO), il sarebbe quell nel caso di Ag strada Palermo

La sconsic dissenso anch stata difesa di simo rigore cl opportunità d zioni, ha prec Valle dei Ter che affinerà $\mathrm{l}$ ricorrerà ancl nessuno e ch tenza dell'en

In Sicilia ce e alcuni mentre per i eletto sindac grande clam giunta spetta è proclamatr

É propric

3. Si tratta ، che ha ottenu serie di delegh

4. Si veda 1 bardo», La Re

5. Si veda! via da Himer: 
i cannoli in mano o il giro del monito non ha impedinazionale del pardal punto di vista unici senatori che

itica e sociale, con ordinario consenso teso rinunciare, inztica modificabilità ro sotto il sole: an), non facevano gli Salvo Lima e Vito

a studiare anche il ombardo, con il suo co molto in comune: a corte di Calogero intino, più volte mito risolta. Hanno in acità di mobilitazionalizzata al di là del lel voto mafioso. Per ) un appello indiretto la dichiarazione del 'eroismo manifestato Vittorio Mangano, a azioni ai pubblici miomuni.

o siciliano, ivi comci di Beppe Grillo, il essere spiegato con ella cosiddetta socie-

laugurata autonomia volazioni fiscali alle ionale dove concenhiappare tutti i fondi co temporale 2007 . critto.

a), La Repubblica-Pa-
Il nuovo Presidente ha faticato non poco per comporre la giunta anche perché ha preteso di coinvolgere due magistrati al di sopra di ogni sospetto. togliendo poltrone a esponenti della coalizione ${ }^{3}$; in compenso sono state moltiplicate le commissioni parlamentari con relative presidenze, segreterie, auto di servizio, autisti etc. e conseguente aumento della spesa.

Nel panorama ordinario costituito dal perdurante buco della Sanità, dalle contestazioni della Corte dei Conti alle spese della Regione e dalla cronica inefficienza dell'elefantiaco apparato regionale, sono apparse sulla stampa nazionale nei primi gionni di luglio alcune dichiarazioni deflagranti dell'Assessore ai Beni culturali (il cuffariano Antonello Antinoro), circa la sua determinazione di cedere a privati per alcuni decenni i siti archeologici più importanti della Sicilia, come la Valle dei Templi di Agrigento (sito UNESCO), il teatro greco di Siracusa, quello di Taormina, ecc. L'obiettivo sarebbe quello di incassare dei canoni ed ottenere la costruzione di opere; nel caso di Agrigento l'Assessore desidererebbe migliorie sul tracciato della strada Palermo-Agrigento e un eliporto; in altri casi alberghi, musei, ecc. ${ }^{4}$

La sconsiderata proposta ha suscitato sconcerto, reazioni allarmate e dissenso anche da parte di esponenti della coalizione di centro-destra, ma è stata difesa dal Presidente che ha cercato di formire rassicurazioni sul massimo rigore che sarebbe stato praticato per l'arruolamento dei privati e sulla opportunita di ascoltare il mondo della cultura. L'Assessore, viste le reazioni, ha precisato ulteriormente il suo pensiero, escludendo al momento la Valle dei Templi e sottolineando che sarà costituito un apposito comitato che affinerà la proposta, che saranno coinvolte banche e fondazioni e che si ricorrerà anche a concorsi di idee ${ }^{5}$. Precisazioni che non hanno rassicurato nessuno e che confermano una certa confusione tra le funzioni di competenza dell'ente pubblico e il ruolo dei privati.

In Sicilia c'è stato anche un turno elettorale che ha riguardato le province e alcuni comuni. Alle elezioni provinciali ha stravinto il centro-destra mentre per i comuni la novità più sorprendente è che Vittorio Sgarbi è stato eletto sindaco di Salemi (piccolo comune in provincia di Trapani) con grande clamore mediatico e dichiarazioni pirotecniche; ha formato una giunta spettacolare in cui c'è anche Oliviero Toscani e Philippe Daverio si è proclamato bibliotecario del Comune. Che dire?

E proprio vero che la realtà, a volte, supera qualunque fantasia.

3. Si tratta di Massimo Russo che ha ottenuto la delega alla Sanita e di Giovanni llarda che ha ottenuto l'Assessorato alla Presidenza. che è una stranezza tutta siciliana, con una serie di deleghe tra cui quella del personale e dei servizi generali.

4. Si veda larticolo di $\mathrm{E}$. Lauria "La Valle ai privati -- Proposta shock della giunta Lombardo", La Repubblica, 3 lug. 2008.

5. Si veda l'articolo di (3. Pipitone "Siti archeologici ai privati. Prime mosse. Antinoro: "il via da Himera e Solunto"», Giornale di Sicilia, 5 lug. 2008. 
11 centro-destra aveva vinto anche le elezioni comunali di Palermo del 2007, confermando il sindaco uscente, l'avvocato ed ex deputato di Forza Italia, Diego Cammarata, a cui il centro-sinistra aveva contrapposto uno sfidante di spessore come Leoluca Orlando. Questi aveva ottenuto un ottimo risultato personale e delle sue liste ma pare che una settimana prima del voto, si sia messa in moto una colossale macchina di compravendita di voti. specie nei quartieri popolari, a cui si sono sommati brogli e scorrettezze di vario genere, che hanno comportato l'incriminazione di alcuni presidenti di seggi elettorali. All'indomani della vittoria elettorale del 2007 il Comune ha aumentato del $75 \%$ la tassa sulla raccolta dei rifiuti solidi urbani (tarsu), senza incentivare per altro la raccolta differenziata che è quasi inesistente, 0 ipotizzare il ciclo integrato del trattamento dei rifiuti. Naturalmente questa iniziativa ha suscitato polemiche e contestazioni. Dopo circa un anno il Comune ha disposto un nuovo balzello per consentire agli automobilisti di circolare nelle zone centrali della città, definite zone a traffico limitato, con varchi videocontrollati, in via di sperimentazione. Questa scelta ha suscitato ulteriori contestazioni perché l'obiettivo finale non sembra quello di ridurre la circolazione delle auto private, incrementando il trasporto pubblico, ma fare cassa con il pagamento delle autorizzazioni e delle eventuali multe La procedura ideata per il rilascio dei pass è stata concepita talmente male che la prima scadenza al 5 maggio 2008 è stata spostata di un mese. I cittadini sono furenti e i più acculturati si sfogano sui gionali, mentre $i$ comitati in difesa dei consumatori e i consiglieri dell'opposizione si sono rivolti alla magistratura ordinaria e amministrativa.

Il Tar ha azzerato l'operato del Comune sostenendo la nullità del provvedimento sulle zone a traffico limitato in assenza di un piano urbano del traffico e il Comune dovrebbe restituire a cittadini e aziende 5 milioni di $€$. Difficile che ciò accada visto che non ci sono nemmeno le somme per il tradizionale festino di Santa Rosalia che si farà a spese della Regione impegnando un milione di euro.

Sulla vicenda delle $\mathrm{Ztl}$ è intervenuta anche l'Autorità di vigilanza sui contratti pubblici che ha avviato un'indagine sull'appalto bandito dal Comune per gestire la vendita dei pass e sull'aggiudicazione dell'appalto. I risultati dell'indagine potrebbero essere canalizzati verso la Corte dei Conti e la Procura della Repubblica.

L'Amministrazione comunale è comunque imperturbabile e cura molto la comunicazione ufficiale, sia attraverso una pubblicistica patinata che attraverso il sito web del Comune. Ci sono molti documenti sui programmi e sugli obiettivi dell'azione amministrativa, ma non sulle procedure, che spesso mettono radicalmente in crisi i percorsi decisionali. Come dimostra la vicenda delle zone a traffico limitato.

\section{Mafia e antir}

La novità de ogni colore esec cortei antimafia, alleanze, alcune

A Palermo, $c$ se delle trasform dere dalla valuta de urbanistiche $\epsilon$ che per molti an tivo sulla città.

Per capire l'i darne la capacità feudi, all'attività droga, alla spart della finanza, ag scurare le intimic "pizzo") nei con risulta quotidianc

Anche altre $r$ mente infiltrate scontro più sang stragi del 1992, Borsellino, da a Bernardo Proven

Nel 1993 in le bombe a Rom $\mathrm{S}$. Giorgio al Ve zionale di Arte 1

L'escalation nelle coscienze, più rilevante fu

6. Nel 1979 veng ic Tcranova. Nel procuratore capo G La Torre a il gener ge Rognoni-La Tor po dell unficio istr dra mobile Beppe Questi e altri delitt ancora in corso, r delitti eccellenti. A te ancora da chiari 
ioni comunali di Palermo del sato ed ex deputato di Forza istra aveva contrapposto uno Questi aveva ottenuto un ottie che una settimana prima del hina di compravendita di voti. mmati brogli e scorrettezze di nazione di alcuni presidenti di settorale del 2007 il Comune Jei rifiuti solidi urbani (tarsu), iziata che è quasi inesistente, o ei rifiuti. Naturalmente questa zioni. Dopo circa un anno il onsentire agli automobilisti di te zone a traffico limitato, con ione. Questa scelta ha suscitainale non sembra quello di riamentando il trasporto pubbliatorizzazioni e delle eventuali rass è stata concepita talmente 8 è stata spostata di un mese. I sfogano sui giornali, mentre i glieri dell'opposizione si sono ativa.

sostenendo la nullità del provssenza di un piano urbano del tadini e aziende 5 milioni di $€$. no nemmeno le somme per il rà a spese della Regione impe-

he l'Autorità di vigilanza sui e sull'appalto bandito dal $\mathrm{Co}-$ aggiudicazione dell'appalto. I lizzati verso la Corte dei Conti

e imperturbabile e cura molto pubblicistica patinata che atIti documenti sui programmi e na non sulle procedure, che si decisionali. Come dimostra

\section{Mafia e antimafia}

La novità del terzo millennio sta nel fatto che tutte le forze politiche di ogni colore esecrano ufficialmente la mafia, partecipano a manifestazioni e cortei antimafia, ma probabilmente ci sono sul campo nuovi attori e nuove alleanze, alcune già emerse, altre ancora da scoprire.

A Palermo, qualunque ragionamento sullo stato del territorio, sulle cause delle trasformazioni urbane e sulle politiche pubbliche non può prescindere dalla valutazione politica ed etica dei soggetti protagonisti delle vicende urbanistiche ed edilizie e delle implicazioni della criminalità organizzata che per molti anni ha detenuto direttamente il potere politico e amministrativo sulla città.

Per capire l'influenza della mafia in Sicilia e a Palermo bisogna ricordarne la capacità di evoluzione e di innovazione, da sistema di controllo dei feudi, all'attività edilizia, al contrabbando di sigarette, al commercio della droga, alla spartizione degli appalti pubblici, alla penetrazione nel mondo della finanza, agli investimenti nella grande distribuzione, senza mai trascurare le intimidazioni e le attività sistematiche di estorsione (il cosiddetto "pizzo") nei confronti di imprenditori, professionisti e commercianti, come risulta quotidianamente dalle cronache.

Anche altre regioni del sud come la Calabria e la Campania sono fortemente infiltrate dalla criminalità organizzata, ma in Sicilia si è verificato lo scontro più sanguinario tra la mafía e pezzi dello stato, culminato nelle stragi del 1992, in cui persero la vita i giudici Giovanni Falcone e Paolo Borsellino, da anni nel mirino dei corlconesi, capeggiati da Totò Riina e Bernardo Provenzano".

$\mathrm{Nel} 1993$ in un delirio di onnipotenza i terroristi corleonesi sbarcano con le bombe a Roma, a Firenze e a Milano e collocano il tritolo nella chiesa di $\mathrm{S}$. Giorgio al Velabro, nell' Accademia dei Georgofili e nella Galleria Nazionale di Arte Moderna, facendo nuove vittime.

L'escalation del terrorismo mafioso provocò finalmente delle reazioni nelle coscienze, nella politica e nelle istituzioni. Sul piano politico l'evento più rilevante fu la relazione conclusiva della Commissione Antimatia della

6. Nel 1979 vengono uccisi if capo della squadra mobile Boris Giuliano e il giudice Cesare Terranova. Nel 1980 vengono uccisi il presidente della Regione Piersanti Mattarella e il procuratore capo Gaetano Costa. Nel 1982 vengono uccisi il segretario regionale del Pci Pio La Torre e il generale Carlo Alberto dalla Chiesa. Dopo questi delitti viene approvata la legge Rognoni-La Torre e istituito il reato di associazione mafiosa. Nel 1983 salta in aria il capo dell ufficio istruzione, Rocco Chinnici. Nel 1985 vengono uccisi il dirigente della squadra mobile Beppe Montana esperto nella caccia ai latitanti e il vice-questore Ninni Cassara. Questi e altri delitti sono stati addebitati ai corleonesi, ma nonostante vari processi, per altro ancora in corso, restano molti misteri sui mandanti, sui killer e sui veri obiettivi di alcuni delitti eccellenti. Anche le circostanze in cui maturarono lo stragi del 1992 sono in gran parte ancora da chiarire. nonostante i numerosi processi a vario stadio di definizione. 
XI legislatura (1992-1994) presieduta da Luciano Violante, nella quale si denunciarono all'unanimità $\mathrm{i}$ rapporti sistematici tra la mafia e ampi settori della politica in Sicilia e a Palermo (Commissione Antimafia, 1993) ${ }^{7}$.

Iniziò anche la stagione degli arresti dei grandi latitanti: il 15 gennaio 1993, nei pressi della circonvallazione, in pieno centro, viene arrestato Totò Riina. ${ }^{\gamma}$ Dopo l'arresto di Riina, la mafia rinuncia allo scontro frontale con lo stato e si inabissa sotto la guida prudente di Bernardo Provenzano, senza mai tralasciare i propri affari".

Il 19 novembre 2002 muore a Roma, nel suo letto, Vito Ciancimino, autore del "sacco" di Palermo e primo politico condannato per mafia, portando con sé molti segreti.

L'11 aprile 2006 viene arrestato anche Provenzano. L'arresto di Provenzano con il suo sterminato patrimonio di «pizzini» (bigliettini) apre nuovi filoni di indagine.

Il 5 novembre 2007 vengono arrestati altri due latitanti di alto rango che gestivano la periferia nord di Palermo: Salvatore e Sandro Lo Piccolo, rispettivamente padre e figlio. Si tratta di una nuova generazione di mafiosi, rifiniti ed eleganti, assai diversi dai «viddani» di Corleone. Dall'archivio dei Lo Piccolo emerge uno spaccato impressionante dei taglieggiati: centinaia di imprese e di attività commerciali, anche le più modeste, pagano il «pizzo»"

A differenza del passato, però, finalmente le associazioni di categoria degli imprenditori e dei commercianti hanno preso ufficialmente posizione contro il taglieggiamento mafioso, invitando gli iscritti a denunciare le ri-

7. «[...] Palermo e la Sicilia restano il territorio di Cosa Nostra. Non a caso nella capitale dell'isola, cuore politico della Regione e punto di snodo delle ingenti risorse finanziarie regionali e statali. Cosa Nostra ha realizzato e manticne una struttura di controllo del territorio non rinvenibile in nessun'altra realtà locale. A Palermo, infatti, contrariamente ad altre loca. lità, dove Cosa Nostra è rappresentata da una sola "famiglia", l'organizzazione mafiosa è presente con una molteplicità di famiglie che si sono suddivise la città in modo da non laksciare scoperto e incontrollato nessun pezzo di territorio». La relazione approvata dalla Commissione parlamentare antimatia della XI legislatura, presieduta da Luciano Violante (da cui è tratto il brano), ha evidenziato con esemplare chiarezza i rapporti antichi ed organici tra mafia e politica in Sicilia e a Palermo.

8. Lo stesso giorno Giancarlo Caselli prende servizio alla Procura di Palermo.

9. Si veda il resoconte stenografico della $16^{\circ}$ seduta della Commissione Antimafia del 14 mag. 2002 nel quale il Procuratore Capo di Palermo, Piero Grasso dice testualmente: «[...] Vorrei rammentare la peculiarità della presenza della criminalità organizzata di lipo mátioso nella provincia di Palermo, ove si trovano la sede riconosciuta dell'organizzazione Cosa Nostra e il centro principale delle più diverse attività criminali organizzate, spesso con estensione nazionale ed internazionale. A proposito dell'attuale par mafiosa il Procuratore Grasso dice: Adesso, attraverso le indagini [...] abbiamo percepito come questa del silenzio, dell'immersione dell'organizzazione, del non commettere più fatti celatanti, sia una vera $\mathrm{e}$ propria strategias.

10. Si veda L.a Repubhlica-Palermo del 27 dic. 2007 chieste estors perdurante co

La rivolta una associazi giunti succes striali e altre mobilitazione nella legalità ne e le scuol questa vera $\mathrm{e}$ tario della fa che ha denun della Kalsa ( tello anti-ma 30 marzo 20 dotti gastron contro la ma dentato, ma s

Il 13 feb della mafia gata storica una fase ero mare in un $p$

\section{I risultati}

Per descr nell'ambito semplificazi

Dal dopo se della citt: munale e de amministrat

I person e Vito Cian dal 1958 al loro, ciò̀ a (1956-62) c vocato guas

11. Si veda sottratto alla $n$ 
iolante, nella quale si la mafia e ampi settori Itimafia, 1993)?

latitanti: il 15 gennaio 0 , viene arrestato Totò o scontro frontale con rdo Provenzano, senza

, Vito Ciancimino, aurato per mafia, portan-

o. L'arresto di Provenbigliettini) apre nuovi

itanti di alto rango che Sandro Lo Piccolo, ri;enerazione di mafiosi, 'orleone. Dall'archivio dei taglieggiati: centijù̀ modeste, pagano il

sociazioni di categoria Ifficialmente posizione itti a denunciare le ri-

a. Non a caso nella capitale genti risorse finanziarie rera di controllo del territorio ontrariamente ad altre local'organizzazione mafiosa d la città in modo da non larelazione approvata dalla eduta da Luciano Violante rapporti antichi ed organi-

ra di Palermo.

missione Antimafia del 14 io dice testualmente: «[...] organizzata di tipo mafioso l'organizzazione Cosa Nonizzate, spesso con estenfiosa il Procuratore Grasso ome questa del silenzio, ii cclatanti, sia una vera $\mathrm{e}$ chieste estorsive e le intimidazioni e minacciandone l'espulsione in caso di perdurante connivenza.

La rivolta contro la mafia è partita circa un anno fa dalle iniziative di una associazione di giovani, denominata Addio pizzo alla quale si sono aggiunti successivamente operatori commerciali, piccoli produttori e industriali e altre associazioni come Libero Futuro. Il cartello ha inaugurato una mobilitazione finalizzata a promuovere la liberta di impresa e i consumi nella legalità e ha prodotto numerose iniziative coinvolgendo la popolazione e le scuole, sia a Palermo che in altre città. Il testimonial più noto di questa vera e propria rivoluzione culturale è Vincenzo Conticello, proprietario della famosa Focacceria S. Francesco, nel centro storico di Palermo, che ha denunciato i taglieggiatori appartenenti alla storica famiglia mafiosa della Kalsa (Spadaro) i quali sono stati condannati anche in appello. Il cartello anti-mafia ha organizzato la prima manifestazione fuori dalla Sicilia il 30 marzo 2008, sbarcando a Roma, a Campo dei Fiori, dove ha offerto prodotti gastronomici siciliani, musiche e spettacoli. Tutte queste iniziative contro la mafia segnano solo l'inizio di un percorso che sarà lungo e accidentato, ma sicuramente sono una novità assoluta di grande rilievo.

Il 13 febbraio 2008 è morto in carcere Michele Greco, il grande capo della matia palermitana condannato a diversi ergastoli, padrone della borgata storica di Ciaculli, immersa in uno splendido territorio agricolo, che in una fase eroica dell'urbanistica palermitana si cercò vanamente di trasformare in un parco agricolo urbano".

\section{I risultati della governance mafiosa}

Per descrivere sinteticamente la natura dei rapporti tra mafia e politica nell'ambito del comune di Palermo bisogna fare alcune schematizzazioni e semplificazioni.

Dal dopoguerra fino alla metà del 1980, esponenti delle famiglie mafiose della citta e della provincia, fanno parte direttamente del consiglio comunale e dell'amministrazione o influenzano da vicino l'attività politica e amministrativa.

I personaggi più famosi sono i democristiani Salvo Lima (andreottiano) e Vito Ciancimino, rispettivamente sindaco e assessore ai Lavori Pubblici, dal 1958 al 1965, ambedue condannati per mafia, anche se tardivamente. A loro, cioè alla governance mafiosa si deve il famigerato piano regolatore (1956-62) che innesco il saccheggio del territorio palermitano e che ha provocato guasti irreparabili nella città, nel centro storico, nel paesaggio.

11. Si veda Cannarozzo (2004) e l'articolo di F. Erbani «Palermo. Cosi muore il parco sottratto alla mafia», La Repubhlica, 19 dic. 2002. 
Le patologie della Palcrmo contemporanea (edificazione a tappeto, squallide periferie pubbliche, degrado del centro storico, mancanza di verde, di scuole, di parcheggi) sono oggettivamente ascrivibili al governo mafioso del territorio esercitato direttamente o attraverso vari prestanome. Sindaci e assessori che si sono succeduti fino al 1985 non avevano in realtà alcuna autonomia dal sistema di potere politico-mafioso.

Il 1985 segna la rottura del rapporto organico tra la mafia e il Comune di Palermo. Viene, infatti, eletto sindaco Leoluca Orlando, democristiano di buona famiglia, non colluso con gli intrallazzi del suo partito, dal quale esce definitivamente nel 1990. Orlando è rimasto in carica fino al 2000 ed è stato il migliore dei sindaci di Palermo, per il contenuto dell'azione politica, per le iniziative urbanistiche e per la capacità amministrativa. Il Comune, infatti, prende posizione per la prima volta contro la mafia e si costituisce parte civile nel maxi-processo (1986) scaturito dalle confessioni del mafioso pentito Tommaso Buscetta ${ }^{12}$.

Orlando avvia anche due iniziative importantissime sotto il profilo urbanistico: la redazione di un piano per il recupero del centro storico (consulenti Leonardo Benevolo e Pierluigi Cervellati, 1988-1993) che ha consentito il restauro di buona parte della città storica e quella di varare un nuovo Prg finalizzato alla riqualificazione della città, molto compromessa da decenni di malgoverno (consulente Cervellati, 1994-2002) (Cannarozzo, 2000; 2004a).

11 territorio comunale di pianura (circa 11.000 ha su 15.888) è infatti soffocato da un'edilizia monolitica che ha sclerotizzato e necrotizzato il suolo e il sottosuolo. Il degrado ambientale si materializza nel grave inquinamento dell'aria e del golfo di Palermo, nella proliferazione di discariche abusive, nel ruolo di fogna a cielo aperto del fiume Oreto, nelle condizioni deprecabili della fascia costiera.

Palermo, per come si è andata configurando nell'età moderna e contemporanea, sembra avere rinnegato le sue origini di città di mare. Con il taglio della Via Maqueda, realizzato alla fine del 1500, si indica una nuova direzione di sviluppo nell'entroterra, verso nord, ruotata di $90^{\circ}$ gradi rispetto alla direttrice prevalente della città medioevale sintetizzata dall'andamento est-ovest del Cassaro. La direzione di sviluppo verso nord non sarà piu smentita nei secoli successivi e renderà sempre più evanescente l'identità marinara delle origini.

La città moderna è cresciuta a dismisura, secondo le discutibili previsioni del piano regolatore del 1962 , e la sua dissennata e spesso illegale attuazione. Sopravvivono miracolosamente alcune porzioni di verde agricolo che hanno conservato alcuni caratteri identitari della Conca d'Oro, ma che

12. Le confessioni di Buscetta (1984), carismatico uomo d'onore, nemico dei corleonesi. al giudice Giovanni Falcone consentirono di istruire il cosiddetto maxi-processo, che porti alla sbarra contemporaneamente circa 500 imputati di associazione matiosa e appioppo una serie di ergastoli ai vertici di Cosa Nostra, tra cui Michcle Greco e Totó Riina. stanno per essere impianti sportivi.

L'espansione u di edifici residenz zioni. Il processo c il territorio agrico nord, travolgendo spansione urbana $\mathrm{c}$ mancano gli insedi ma anche attrezzat centri di rottamazic

Al sistema resic popolare, realizzati degli anni 60 . Ion sporto pubblico, pa parcheggi, nei qual

Dai dati in poss che siano stati real sorbitante rispetto caso di ricordare ch realtà utilizzati più

La storia urbanis lizzate con la conni zioni e di tecnici co dell'Addaura, local di Mondello, comp villeggiatura; le lott dei Colli a ridosso le lottizzazioni che

13. Se quantifichiamo adotlando lo standard $68.672 .000 \mathrm{mc}$. Anche certa percentuale ad attr cune centinatia di milion che non si i comunque occupate, oltre la capacit

14. La politica degli a attività e funzioni pubbl fatto ricorso e vi continu prattutto la Regione, che cortificati per civile abita residenziali per funzioni sulle destinazioni d'uso d agli abitanti e contribuiso della città 
Conca d'Oro, fino ad arrivare alla edificazione scandalosa di Pizzo Sella, avviata dal costruttore Notaro, cognato da Michele Greco, alla fine degli anni '70 (battezzato dai mass media come la «Collina del disonore») $)^{15}$. Si tratta per lo più di énclaves recintate, dai nomi suggestivi, abitate da famiglie benestanti, non si sa quanto consapevoli dell'origine più o meno criminale del proprio habitat.

In realtà queste edificazioni non dovrebbero essere chiamate lottizzazioni, perché non ne hanno la natura giuridica. Si tratta di iniziative edificatorie illegittime, sia dal punto di vista delle procedure, che dal punto di vista dei contenuti progettuali. Siamo in presenza di frazionamenti di aree destinate dal Prg del 1962 a verde agricolo, nel quale si prevedeva però un indice di fabbricabilità di $0,20 \mathrm{mc} / \mathrm{mq}$ per costruire fabbricati connessi all'uso agricolo del suolo. I frazionamenti sono serviti invece per realizzare ampi comparti di residenze unifamiliari più o meno lussuose, serviti da strade private, con accessi controllati, in cui mancano del tutto le attrezzature che dovrebbero essere inserite nelle lottizzazioni regolari.

Il tutto è accaduto con la connivenza del Comune e dell'Assessorato regionale al Territorio e Ambiente a cui spetta la vigilanza sull'operato dei comuni. I procedimenti attraverso cui sono state realizzate tutte queste costruzioni meriterebbero di essere chiariti attraverso un'apposita ricerca sui documenti tecnici, sugli elaborati progettuali, sugli atti amministrativi. Il Comune, in occasione della redazione del nuovo piano regolatore ritenne di risolvere il problema alla radice definendo queste zone come zone $B$ sature, ma l'occhiuta Regione, consapevole delle magagne di base, ha preteso che queste zone fossero classificate $C$ e che venissero trovate le aree da destinare a servizi e attrezzature secondo gli standard di legge. Come, non si sa.

La disseminazione di ville e villette si è manifestata anche nelle altre zone di pianura del territorio comunale, nella Valle dell'Oreto e nella periferia sud-orientale costellata dalle borgate controllate dalla mafia palemitana: Ciaculli, Santa Maria di Gesù, Croceverde-Giardini. Si tratta pero di iniziative più limitate, sfacciatamente abusive, nel senso che non si sono nemmeno cercate coperture pseudo-legali; le costruzioni, molto più modeste, sono destinate a fasce sociali meno abbienti e spesso sono realizzate in proprio dagli interessati. Queste iniziative non sono minimamente paragonabili all'edificazione a tappeto della Piana dei Colli, né per l'ampiezza del territorio interessato né per la tipologica delle abitazioni.

La zona sud-orientale di Palermo è stata, infatti, quella meno investita dall'urbanizzazione e da trasformazioni irreversibili ed è quella che ha conservato più delle altre ampie zone agricole destinate prevalentemente alla coltivazione del mandarino tardivo e altri alberi da frutta.

15. Si veda l'articolo di R. Arena «Pizzo Sella, prescrizione per 3 imputati. Ma dovranno pagare i risarcimenti», Giomale di Sicilia, 17 dic. 2002
C'è il ragionevole lungo periodo dalla fa sivo nell'incentivare 1 per preservare l'assett sedeva ampie propriet l'inimmaginabile vice molto lontano dalle zo

A partire dagli an settori di investimentc ciali. Le indagini scatı mafia, Antonino Giuf tecnici, costruttori e at falsificazione delle $\mathrm{pr}$ muni e hanno portato costiera che va da Can

Per quanto riguard indagini giudiziarie in nopolizzare la realizz percorso che prevede scossione di tangenti appalti e ai subappalt venzano, che dopo le imprenditoriale della centro commerciale a volte per infiltrazio L'iniziativa è stata $n$ Campanella, moderno messo in luce le com società romane $\mathrm{e}$ il $\mathrm{cl}$

16. Recentemente il $\mathrm{Co}$ giorni, ma i proprietari. mento da parte de Comun la di risolutivo. Si veda l' mese per lasciare le ville"

17. Antonino Giuffè, vi autorevoli pentiti. Da que lano delle connessioni tra

18. Si prevedeva la re con cinema multisala, dell operarione è la Ass nella, devoto di Provenz manifestazioni contro la blicati su La Repubblica di Palermo, e gli articoli 


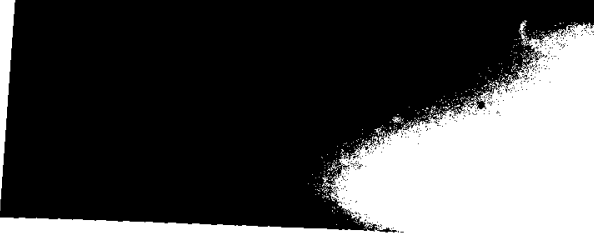

alosa di Pizzo Sella, Jreco, alla fine degli a del disonore») $)^{15}$. Si stivi, abitate da famiine più o meno crimi-

\section{: chiamate lottizzazio-} di iniziative edificatoche dal punto di vista inamenti di aree destirevedeva però un indirricati connessi all'uso ce per realizzare ampi uose, serviti da strade utto le attrezzature che

ze dell'Assessorato re;ilanza sull'operato dei lizzate tutte queste coun'apposita ricerca sui i atti amministrativi. II no regolatore ritenne di ne come zone $B$ sature, di base, ha preteso che ovate le aree da destinage. Come, non si sa. estata anche nelle altre dell'Oreto e nella perite dalla mafia palermiardini. Si tratta però di senso che non si sono zioni, molto più modebesso sono realizzate in minimamente paragoné per l'ampiezza del oni.

quella meno investita ed è quella che ha cone prevalentemente alla utta.

3 imputati. Ma dovranno
C'è il ragionevole sospetto che la mafía palermitana, capeggiata per un lungo periodo dalla famiglia di Michele Greco, abbia avuto un ruolo decisivo nell'incentivare la direttrice di espansione della città verso nord anche per preservare l'assetto delle borgate agricole sud-orientali, nelle quali possedeva ampie proprietà e dove si svolgevano varie attività dei clan. Infatti, l'inimmaginabile vicenda di Pizzo Sella, si svolge all'altro capo della città, molto lontano dalle zone di immediata influenza della famiglia ${ }^{16}$.

A partire dagli anni ' 90 l'imprenditoria mafiosa si cimenta con nuovi settori di investimento: gli insediamenti turistici e i grandi centri commerciali. Le indagini scaturite dalle confessioni di uno dei più illustri pentiti di mafia, Antonino Giuffrè, hanno evidenziato la complicità tra boss, uffici tecnici, costruttori e amministratori; hanno verificato lo stravolgimento e la falsificazione delle previsioni urbanistiche dei piani regolatori di vari comuni e hanno portato al sequestro di alcuni complessi turistici sulla fascia costiera che va da Campofelice a Cefalít ${ }^{17}$.

Per quanto riguarda i centri commerciali, a Palermo e provincia ci sono indagini giudiziarie in corso sull'interesse di alcune famiglie mafiose a monopolizzare la realizzazione di grandiose strutture di vendita, attraverso un percorso che prevede l'intermediazione sull'acquisizione dei terreni, la riscossione di tangenti da parte dei gruppi investitori, la partecipazione agli appalti e ai subappalti. Il tutto avallato dalla benedizione di Bernardo Provenzano, che dopo le stragi del 1992 è stato l'ispiratore della nuova politica imprenditoriale della mafia. Esemplare è il caso del progetto di un megacentro commerciale a Villabate, comune alle porte di Palermo, sciolto due volte per infiltrazioni mafiose e dominato dalla famiglia Mandalà. L'iniziativa è stata minutamente descritta nelle confessioni di Francesco Campanella, moderno mafioso, politico e consulente finanziario, che hanno messo in luce le complicità tra tecnici palermitani, politici di tutti i partiti, società romane e il clan mafioso dei Mandalà (2000-2005) ${ }^{1 \mathrm{t}}$.

16. Recentemente il Comune ha inviato ai proprietari le ordinanze di sgombero entro 30 giorni, ma i proprietari, forti di alcune sentenze che hanno sancito it loro diritto al risarcimento da parte de Comune, intendono resistere. E oltre modo probabile che non accada nutla di risolutivo. Si veda l'articolo di S. Scarafia, «Ultimatum alle famigtie di Pizzo Sella "Un mese per lasciare le ville"», La Repuhblica-Palermo, 2 lug. 2008.

17. Antonino Ginffé, vice di Provenzano, è. dopo Tommaso Buscetta, il secondo dei mafiosi autorevoli pentiti. Da quello che si è appreso dai giomali, le sue confessioni (2002-2003) parlano delle connessioni tra la mafic e nuovi referenti politici, stabilite dopo le stragi del 1992.

18. Si prevedeva la realizzazione di un centro commerciale Auchan da 200 milioni di $€$. con cinema multisala, ristoranti e negori. esteso 14 ha. La socictà romana incaricata delloperazione è la Asset Development, presieduta da Paolo Marussig. Francesco Campanella, devoto di Provenzano e amico di Cuffaro, è un nuovo tipo di mafioso che organizza manifestazioni contro la mafia e frequenta magistrati inquirenti. Si vedano gli articoli pubblicati su La Repubblica dell's mar. 2006. cronaca nazionale e pagine ll e lll della cronaca di Palermo, e gli articoli di V. Fagone. «Matia e Affari. Retata con 18 arresti» e L. Gargano 
Il progetto di Villabate entra però in competizione con un'altra iniziativa analoga, promossa nello stesso periodo a Palermo nella zona di Brancaccio, dal boss locale, il medico Giuseppe Guttadauro, e appoggiata da esponenti dell'amministrazione comunale di centro-destra ${ }^{19}$. Nonostante il clamore della vicenda e gli espliciti interessi mafiosi, il progetto è andato avanti e stanno per cominciare i lavori, mentre l'ambizioso programma di Villabate è stato fortemente ridimensionato ${ }^{211}$.

\section{Il centro storico}

Le condizioni del centro storico (circa 30.000 abitanti su 250 ettari) sono ancora oggi molto diversificate e manifestano forti contraddizioni; il recupero si è realizzato a "macchia di leopardo" ed è possibile incontrare a poca distanza edifici ben restaurati e rovine sommerse dalla spazzatura; percorrere gli ambienti vivi e chiassosi dei mercati all'aperto e zone di macerie disabitate.

Il centro storico di Palermo continua e esprimere una doppia identità; quella aulica derivante dai valori paesaggistici, dall'architettura arabonormanna, dalla croce di strade che ha disegnato i quattro mandamenti, dal grande patrimonio edilizio barocco e quella popolare conferita dal sistema dei grandi mercati storici all'aperto (Capo, Ballarò, Vucciria) caotici e pittoreschi di giorno, spettrali $\mathrm{e}$ inquietanti di notte. Essi presentano ancora oggi una notevole concentrazione di attività commerciali ed esprimono una tradizione culturale più mediorientale che europea?"

Si tratta di mercati prevalentemente alimentari, che tra l'altro commerciano ancora oggi produzioni rare ottenute da attività agricole residuali a conduzione familiare, vicine alla città. Essi sono caratterizzati da un'economia fragile, con una forte percentuale di abusivi e svolgono la loro attività in ambienti urbani fortemente degradati. La loro presenza e la necessità di

«II centro commenciale della discordia», Gionnale di Sicilia, 8 mar. 2006: R. Arena, «Mandala dietro l'affare», Giomale di Sicilia, 14 apr. 2006.

19. Brancaccio è un quartiere periferico a sud di Palemo, ad alta densità mafiosa, dove è stato ucciso il 15 settembre 1993, il parroco, Padre Pino Puglisi. I soggetti promotori del centro commerciale sono il colosso francese (arrefour con due partners (il consorzio Panormus e Collegno 2000). L'iniziativa parte nel maggio 2002, quando la Conferenza di servizi indetta dall'ufficio dello Sportello unico del Comune di Palermo approva il megaprogetto che investe un area di 32 ettari. Intercettazioni telefoniche dei Ros famno emergere if coinvolgimento dell'assessore comunale Domenico Miceli (anch'egli medico), che verra arrestato. Si veda l'anticolo di R. Arena, (Politici, primari, piano regolatore. Colloqui tra un boss e un medicos, Giornale di Sicilia, 18 dic. 2002.

20. La commissione prefettizia insediata al Comune, nella primavera del 2006 ha ridotto l'estensione dell'area commerciale a soli 4 ha

21. Fatte le debite proporzioni, è straordinaria la somiglianza di questi ambienti con alcune aree commerciali del centro storico di Istanbul. salvaguardarli senza al stituisce un'ulteriore a perché genera contem! e illegalità, abbondanz deserti notturni.

Già nell' 800 , il ce mente da piccola bor: sovraffollamento e da demie di colera. La no propri fastosi palazzi più consoni alle propr già iniziata; l'assenza maniera drammatica $i$

Durante la II guer bombardamenti, le cl comincia l'esodo degl quartieri popolari e qu espansione; quest'ulti piano regolatore allor tico tessuto urbano (C provocato la rarefazi scenza del patrimonic

Il centro storico c più importanti, disloc principale e una certa le-espositivo. che po zione del patrimonio abbandonato o sotto-

La chiesa e gli o proprio ruolo e delle frono assistenza spec zioni di assicurare ur

Il centro storico gran numero di extra ci fortemente degrac loro presenza ha co phone center e di att gianali etnici.

Si verificano pe della città storica (A ze di sgombero, che 
one con un'altra iniziativa nella zona di Brancaccio, a appoggiata da esponenti 19. Nonostante il clamore rogetto è andato avanti e io programma di Villabate

J abitanti su 250 ettari) so) forti contraddizioni; il reed è possibile incontrare a ommerse dalla spazzatura; ati all'aperto e zone di ma-

imere una doppia identità; ci, dall'architettura arabos i quattro mandamenti, dal jolare conferita dal sistema arò, Vucciria) caotici e pittte. Essi presentano ancora nmerciali ed esprimono una $\mathrm{ea}^{21}$.

ari, che tra l'altro commerattività agricole residuali a caratterizzati da un'econoi e svolgono la loro attività o presenza e la necessità di

ia. 8 mar. 2006; R. Arena, «Man

10, ad alta densità mafiosa, dove è Puglisi. I soggetti promotori del on due partners (il consorzio $\mathrm{Pa}$ 002, quando la Conferenza di serdi Palermo approva il megaprogetniche dei Ros fanno emergere il eli (anch'egli medico), che verrà piano regolatore. Colloqui tra un

ella primavera del 2006 ha ridotio ianza di questi ambienti con alcu- salvaguardarli senza alterame la natura popolare, invasiva ed anarchica, costituisce un'ulteriore articolazione problematica dell'uso della città storica, perché genera contemporaneamente vitalità e degrado, pregio merceologico e illegalità, abbondanza di prodotti e montagne rifiuti, congestione diurna e deserti notturni.

Già nell' 800 , il centro storico è un'area degradata, abitata prevalentemente da piccola borghesia e proletariato, caratterizzata da un pericoloso sovraffollamento e da precarie condizioni igieniche che alimentano le epidemie di colera. La nobiltà, in piena crisi economica, ha già abbandonato $i$ propri fastosi palazzi e la nascente borghesia preferisce insediarsi in luoghi più consoni alle proprie aspirazioni. La decadenza del patrimonio edilizio è già iniziata; l'assenza di manutenzione da parte della proprietà accelererà in maniera drammatica i processi di degrado degli edifici.

Durante la II guerra mondiale il centro storico subisce una valanga di bombardamenti, le cui conseguenze sono ancora visibili. Dopo la guerra comincia l'esodo degli abitanti: quello coatto dei ceti meno abbienti verso $i$ quartieri popolari e quello della piccola e media borghesia verso le zone di espansione; quest'ultima è spinta ad allontanarsi anche dalle previsioni del piano regolatore allora vigente, che prevedeva la totale distruzione dell'antico tessuto urbano (Cannarozzo, 2000). L'allontanamento degli abitanti ha provocato la rarefazione delle attività economiche e ha aggravato la fatiscenza del patrimonio edilizio residenziale.

Il centro storico continua a ospitare le attività e le funzioni istituzionali più importanti, dislocate in splendidi edifici ubicati sulle piazze e sulle vie principale e una certa quantità di funzioni culturali, per lo più di tipo museale-espositivo, che potrebbero ulteriormente espandersi attraverso l'utilizzazione del patrimonio edilizio monumentale attualmente disponibile e spesso abbandonato o sotto-utilizzato (Orlando, 2006).

La chiesa e gli ordini conventuali, pur nella progressiva riduzione del proprio ruolo e delle proprie risorse, costituiscono ancora un presidio e offrono assistenza specie alle comunità immigrate, ma non sono nelle condizioni di assicurare una adeguata manutenzione dei propri beni.

Il centro storico è ormai popolato a chiazze e tra gli abitanti ci sono un gran numero di extra-comunitari, di cui molti clandestini, che abitano edifici fortemente degradati correndo gravi rischi per la propria incolumità. La loro presenza ha comportato l'apertura di un numero sempre crescente di phone center e di attività commerciali connesse a prodotti alimentari o artigianali etnici.

Si verificano periodicamente crolli di edifici pericolanti in varie zone della città storica (Albergheria, Capo, Vucciria) e si susseguono le ordinanze di sgombero, che mettono sulla strada decine di famiglie; l'ultimo cedi- 
mento di una palazzina, nei pressi della cattedrale, ha provocato la morte di un giovane operaio di ventisette anni ${ }^{22}$.

Nel centro storico vi sono ancora grosse sacche di marginalità e di degrado sociale con manifestazioni di grave devianza di cui è vittima predestinata l'infanzia, spesso con la connivenza delle famiglie; vi si concentrano anche una serie di attività illecite come macellazione clandestina, ricettazione, spaccio di droga e altre forme di utilizzazione impropria del patrimonio edilizio anche monumentale. Il centro storico costituisce anche un serbatoio inesauribile per furti di opere d'arte e asportazioni sistematiche di arredi, pavimentazioni storiche, ecc.

A questa grave situazione di disagio sociale fa da contraltare il ritorno di pochi abitanti culturalmente ed economicamente selezionati e l'apertura, specie lungo le vie e le piazze principali, di ristoranti, pub, piani bar, enoteche, ecc. che scatenano una frenetica vita notturna ed entrano in conflitto con i pochi residenti superstiti.

\section{Il processo di recupero del centro storico}

Dopo aver richiamato alla memoria l'identità, le condizioni e le problematiche emergenti nel centro storico, passiamo ad esaminare lo stato di attuazione dei piani urbanistici finalizzati al recupero e le politiche pubbliche attuali.

Come è noto a Palermo ha operato dal 1993 il Piano particolareggiato esecutivo per il recupero del centro storico, denominato comunemente Ppe. $^{23}$. La filosofia di fondo del Ppe si basava sullo studio della storia della città e dei suoi processi di trasformazione coma matrici delle scelte progettuali, ispirate prevalentemente alla conservazione del patrimonio edilizio e degli spazi inedificati cosi come consegnati dal passato. Il Ppe ha consentito in maniera abbastanza efficace la ricostruzione dell'edilizia residenziale minore e di quella palaziale, non troppo devastata, mentre non ha avuto successo il ripristino filologico dei palazzi nobiliari semidistrutti come $\mathrm{Pa}$ lazzo Geraci o Palazzo Bonagia.

Nello stesso anno dell'approvazione dei piani da parte della Regione, nella legge regionale finanziaria ( Ir 15/1993) vengono stanziati 170 miliardi di vecchie lire per il recupero del centro storico. Dopo qualche mese, nella

22. Si veda l'articolo di M. Lorello, «Allarme centro storico - a rischio crollo 180 edificir, La Repubblica-Palermo, 11 dic. 2007.

23. In verità il Ppe copre circa i due terzi del centro storico; la rimanente parte, infatti, era sta normata contestualmente dal Piano particolareggiato per l'Albergheria (coordinatore Trombino) e da piani esecutivi redatti dalla Società Italter. Tutti questi piani furono approvati dalla Regione nel 1993 e non sono stati piu sottoposti ad alcuna revisione. Il Comune ha omologato i piani di altra paternita alla normativa del Ppe; pertanto quando si parla di recupero del centro storico di Palermo si evoca solamente il Ppe (Cannarozzo, 1996).
Ir 25/1993 (agli a: buti finanziari ai 1 accedere mediants buti vengono ero६ interessi per le sin ti sei bandi con un

Il processo di ciarne un sintetic zioni e i nodi irris condizione necess damente i risultati mettendo in camp dinate, soggetti pu

I protagonisti c che hanno utilizza il Comune stesso, denza ai Beni cult scelti opportunam stati individuati e Pertanto c stato $\mathrm{n}$ dilatazione dei ten lentemente disfun to in porto il coinv

Il Comune, all senza avere dati s getti da coinvolge ve e inadeguate hanno impedito la

Solo nel quint mente gli interven difici particolarm tributi le imprese non fare un cenn giudiziarie e svari sione o addirittura

Bisogna ricon 2001. pur tra var centro storico, po complesso di S. A del Noviziato dei co, promovendo avviando il recup piccole aree di pa 
ocato la morte di

rginalità e di deè vittima prede$\because$ vi si concentraslandestina, ricetupropria del patri;tituisce anche un mi sistematiche di

raltare il ritomo di onati e l'apertura, , piani bar, enotentrano in conflitto

dizioni e le probleinare lo stato di atpolitiche pubbliche

10 particolareggiato nato comunemente lio della storia della delle scelte progetatrimonio edilizio e Il Ppe ha consentiedilizia residenziale entre non ha avuto nidistrutti come $\mathrm{Pa}$ -

arte della Regione, anziati 170 miliardi qualche mese, nella

ischio crollo 180 edifianente parte, infatti, era sergheria (coordinatore ti piani furono approvaevisione. Il Comune ha quando si parla di recuzzo, 1996).
Ir 25/1993 (agli artt. 124-125-126 e 127) si dispone l'erogazione di contributi finanziari ai privati per il recupero di edilizia residenziale a cui si potrà accedere mediante bandi e regolamenti messi a punto dal Comune. I contributi vengono erogati in conto capitale per le parti condominiali e in conto interessi per le singole unità immobiliari. Dal 1993 a oggi sono stati emanati sei bandi con un successo sempre crescente.

Il processo di recupero è iniziato e dopo quindici anni è possibile tracciarne un sintetico bilancio, evidenziando i punti di criticità, le contraddizioni e i nodi irrisolti. Avere avuto un buon piano è stata sicuramente una condizione necessaria ma certamente non sufficiente per raggiungere rapidamente i risultati auspicati; gli obiettivi dei piani si realizzano faticosamente mettendo in campo politiche pubbliche, risorse economiche, strategie coordinate, soggetti pubblici e privati (Cannarozzo, 2004b; Orlando, 2004).

I protagonisti del recupero, sono stati finora i privati singoli e associati, che hanno utilizzato gli appositi canali fmanziari previsti dalla Ir n. 25/1993, il Comune stesso, lo lacp, l'Università, l'Opera universitaria, la Soprintendenza ai Beni culturali. Allo Iacp sono stati assegnati immobili non sempre scelti opportunamente: in luogo di indicare edifici vuoti e degradati sono stati individuati edifici dove erano in corso interventi privati di restauro. Pertanto è stato necessario rivedere alcune assegnazioni con una notevole dilatazione dei tempi degli interventi. Per svariati motivi riguardanti prevalentemente disfunzioni sulle assegnazioni degli immobili non è finora andato in porto il coinvolgimento delle cooperative edilizie.

Il Comune, all'inizio, ha predisposto l'erogazione di contributi ai privati senza avere dati sulla proprietà edilizia nel centro storico e quindi sui soggetti da coinvolgere, tramite bandi con condizioni particolarmente restrittive e inadeguate che hanno prodotto la dispersione degli interventi e che hanno impedito la riqualificazione di comparti significativi.

Solo nel quinto bando sono stati modificati i criteri privilegiando finalmente gli interventi su intere unita edilizie, dando priorità al recupero di edifici particolarmente degradati e includendo tra i soggetti ammessi ai contributi le imprese edili e le società immobiliari. A questo punto non si può non fare un cenno sulla natura spesso equivoca di tali soggetti: indagini giudiziarie e svariati processi hanno evidenziato ancora una volta la collusione o addirittura la coincidenza con esponenti di Cosa Nostra.

Bisogna riconoscere che l'amministrazione Orlando, in carica fino al 2001, pur tra varie contraddizioni, ha dato un forte input al recupero del centro storico, portando in appalto il restauro di edifici pubblici come il complesso di S. Anna, ora adibito a Galleria d'Arte Moderna, il complesso del Noviziato dei Crociferi, dove ha sede l'Assessorato per il Centro storico, promovendo la riutilizzazione culturale del complesso dello Spasimo, avviando il recupero dell'area costiera antistante il Foro Italico, realizzando piccole aree di parcheggio alberate (Olivella) e qualche giardino (via Allo- 
ro). Probabilmente l'errore maggiore fatto dall'amministrazione Orlando è stato quello di considerare il Ppe un punto di arrivo, che non si poteva mettere in discussione e di non avere valutato la necessità di varare politiche pubbliche variabili al variare della situazione. Da un monitoraggio intelligente del processo di recupero sarebbe potuta scaturire l'opportunità di introdurre nuove previsioni come l'implementazione e la riqualificazione degli spazi pubblici in luogo di una rassicurante ricostruzione del tessuto edilizio distrutto da eventi di varia natura. Cosi come sarebbe stato necessario moltiplicare l'intervento pubblico residenziale per abbattere la speculazione immobiliare in ascesa. Dal Ppe, come gli stessi autori prevedevano, sarebbero potuti scaturire una serie di piani di settore tra loro integrati, da concertare con gli altri enti competenti e con le categorie produttive ${ }^{2+}$.

I numerosi restauri attuati e in corso di attuazione si manifestano come la somma un po' casuale di interventi singoli, realizzati sulle piazze e sulle vie di maggior pregio. Il recupero della citta storica, partito con lentezza e con un notevole spreco di risorse finanziarie erogate "a pioggia"; ha avuto recentemente una certa accelerazione, non esente da disfunzioni di vario genere, quando vari soggetti economici, impossibilitati a costruire altrove per mancanza di aree disponibili, hanno capito che l'investimento nel centro storico poteva diventare molto conveniente.

In pochi anni si è creato un mercato immobiliare prima inesistente e i prezzi degli immobili sono cresciuti enormemente. Ciò è testimoniato anche dall'apertura di numerose agenzie immobiliari e dal numero sempre crescente di pagine dei quotidiani destinate alla compravendita di immobili nel centro storico, che fino a dieci anni fa non era minimamente preso in considerazione.

Naturalmente tutto ciò prelude alla cacciata degli abitanti meno abbienti, siano essi indigeni o extra-comunitari, alla sparizione dei piccoli esercizi commerciali e artigianali: in una parola, a un ricambio radicale di abitanti e di attività al di fuori di qualsivoglia controllo pubblico.

Nel settembre 2003 i piani particolareggiati per il recupero del centro storico hanno completato il loro decennio di vita ma l'Amministrazione comunale (sindaco Cammarata) non ha preso alcuna iniziativa organica al riguardo.

Il Comune ha approvato recentemente il sesto bando per il finanziamento di privati, imprese e società immobiliari per una spesa di circa

24. Proviamo a elencarne alcuni: il piano dell'edilizia residenziale pubblica, il piano delle sedi per attività culturali ed espositive, il piano della ricettività alberghiera, il piano degl spazi pubblici e del verde. il piano dei parcheggi, il piano delle attività commerciali, ecc. (Cannarozzo, 1994).
$20.000 .000 \mathrm{di} €^{25} .11$ regolam da eseguire su una intera uni consentito ad alcuni sogget lazzi monumentali, a poco $\mathrm{f}$ gliaia di euro per i restauri effettuare con contributi pu 130. L'Amministrazione co lizzazione di alberghi di lus: co, predisponendo apposite riate proteste, anche per la $r$ potenzialità turistiche della fico delle facciate degli edi ziato con 30 milioni di $€$ da accantonato ${ }^{28}$.

Con le elezioni comuna marata, con delega al centr personalità di rilievo, con dell'intensificarsi dei crolli edifici pericolanti dal quale interventi più o meno urgen condizioni di degrado pre vengono infatti individuati mente per evitare il totale essere anche dilazionato. D ricolanti sono abitati e al C famiglie per notificare l'or

25. Delibera di CC 1.51 del 2 impiegavano 90 miliardi di $£$ di di $€$ di fondi comunali.

26. Uno dei soggetti più attivi gona, di nobilc famiglia, che ha in via Garibaldi, Palazzo Bugio proprietaria, un anziana nobildo sono stati ubicati uftici comunali

27. Gli edifici individuati son Cassa di Risparmio, Palazzo Buf teleone. Immobile in via Cassar realizzazione di un albergo a $\mathrm{Pa}$ protestato le associazioni Salvar e Italia Nostra.

28. La delibera di Giunta con dell'intervento

29. Si deve a Nino Scimemi, la formazione delle linee' guida poche iniziative felici promoss molto innovativa per il recupero 
zzione Orlando è on si poteva meti varare politiche aitoraggio intelliopportunità di inqualificazione de1e del tessuto ediie stato necessario re la speculazione evedevano, sareb, integrati, da conduttive ${ }^{24}$.

manifestano come sulle piazze e sulle rtito con lentezza e pioggia"; ha avuto lisfunzioni di vario a costruire altrove vestimento nel cen-

rima inesistente e $\mathrm{i}$ j è testimoniato andal numero sempre ivendita di immobili inimamente preso in

itanti meno abbienti, dei piccoli esercizi radicale di abitanti e

recupero del centro a l'Amministrazione niziativa organica al

o per il finanziamenuna spesa di circa

e pubblica, il piano delle berghiera, jl piano degli ittività commerciali, ecc.
20.000 .000 di $€^{25}$. Il regolamento di attuazione destina $i$ fondi solo ad opere da eseguire su una intera unità edilizia. Questa misura, in sé ragionevole, ha consentito ad alcuni soggetti che si erano accaparrati per tempo interi palazzi monumentali, a poco prezzo, di ricevere contributi di centinaia di migliaia di euro per $\mathrm{i}$ restauri ${ }^{26}$. Attualmente $\mathrm{i}$ cantieri aperti per restauri da effettuare con contributi pubblici derivanti da tutti $i$ bandi emanati, sono 130. L'Amministrazione comunale attuale si è mobilitata anche per la realizzazione di alberghi di lusso in molti edifici monumentali del centro storico, predisponendo apposite varianti urbanistiche, che hanno suscitato svariate proteste, anche per la mancanza di uno studio che verifichi le effettive potenzialità turistiche della città ${ }^{27}$. Ha puntato anche sul restauro scenografico delle facciate degli edifici sugli assi principali della città storica, finanziato con 30 milioni di $€$ dalla Regione, ma l'intervento sembra essere stato accantonato $^{28}$.

Con le elezioni comunali del 2007 entra a fare parte della Giunta Cammarata, con delega al centro storico, Nino Scimemi, ex direttore regionale, personalità di rilievo, con grande esperienza amministrativa ${ }^{29}$. A causa dell'intensificarsi dei crolli il nuovo assessore avvia un censimento degli edifici pericolanti dal quale risulta che ben 1.300 edifici hanno bisogno di interventi più o meno urgenti di ristrutturazione. Tra questi, 500 presentano condizioni di degrado preoccupante anche se leggermente diversificato; vengono infatti individuati 180 edifici da mettere in sicurezza immediatamente per evitare il totale cedimento e 320 edifici su cui l'intervento può essere anche dilazionato. Dal censimento risulta che 67 edifici tra quelli pericolanti sono abitati e al Comune si pone un triplo problema: rintracciare le famiglie per notificare l'ordinanza di sgombero, assicurare un ricovero per i

25. Delibera di CC n. 51 del 29 mar. 2006. I primi quattro bandi (1995, 1997, 1998, 1999) impiegavano 90 miliardi di $£$ di fondi regionali; il quinto bando (2002) impiegava 15 milioni di $\epsilon$ di fondi comunali.

26. Uno dei soggetti più attivi e più favoriti è stato un certo Roberti Bilotti Ruggi d'Aragona, di nobile famiglia, che ha comprato, tra gli altri, uno splendido edificio settecentesco in via Garibaldi, Palazzo Bugio Aragona di Villafiorita, venduto a prezzo stracciato dalla proprietaria, un'anziana nobildonna, da tempo residente fuori Palermo. In questo edificio sono stati ubicati uffici comunali.

27. Gli edifici individuati sono Palazzo Di Napoli, Palazzo di Costantino, Palazzo dell'ex Cassa di Risparmio, Palazzo Bufera. Palazzo Piraino, Palazzo ex Satris, Edificio in via Monteleone, Immobile in via Cassari, angolo via Chiavettieri, Edificio in via Butera. Contro la realizzazione di un albergo a Palazzo Villafranca, attualmente di proprietà della Curia hanno protestato le associazioni Salvare Palermo, Legambiente, Fai. Comitato per il centro storico e Italia Nostra.

28. La delibera di Giunta comunale n. 9 del 27 gen. 2006 dichiara la "priorità assoluta" dell' intervento.

29. Si deve a Nino Scimemi, allora Direttore dell' Assessorato regionale ai Beni Culturali, la formazione delle Linee guida del Piano territoriale paesistico regionale (1999): una delle poche iniziative felici promosse dalla Regione Siciliana e l'emanazione di una circolare molto innovativa per il recupero dei centri storici (n. 3/2000). 
nuclei familiari da allontanare e trovare i proprietari degli immobili a cui intimare l'inizio dei lavori di ristrutturazione. Poiché rintracciare e mobilitare la proprietà edilizia, quasi sempre estremamente frazionata è un obiettivo difficilmente raggiungibile in tempi brevi, il Comune ha deciso di attuare le opere urgenti "in danno" riservandosi di studiare un percorso legale per acquisire la proprietà degli edifici pericolanti che saranno ristrutturati in sostituzione dei proprietari inadempienti.

Ai senza casa, tra cui molte famiglie di extra-comunitari, il Comune ha offerto dei container in periferia, ma l'offerta non ha convinto la maggior parte degli interessati che non vorrebbero lasciare le abitazioni. In difesa degli abitanti colpiti dalle ordinanze di sgombero è sceso in campo il Comitato di Lotta per la casa, già attivo da alcuni anni, che ha organizzato manifestazioni e mobilitazioni, proponendo una verifica sull'uso degli immobili confiscati alla mafia e forme di auto-recupero da parte degli abitanti, con l'assistenza tecnica del Comune ${ }^{30}$.

Questa vicenda da il segno di quanto sia problematico il percorso verso il recupero del centro storico e come questo si intrecci con il problema abitativo della popolazione più disagiata.

L'amministrazione Cammarata non ha finora tracciato una politica organica per accelerare il recupero, non ha previsto l'aggiomamento della strumentazione urbanistica, non ritiene necessario realizzare una quota significativa di edilizia residenziale pubblica, che potrebbe contribuire a risolvere il problema abitativo e contemporaneamente calmierare il mercato immobiliare, non si è pronunciata sulle attività $\mathrm{e}$ sulle funzioni da privilegiare nel centro storico con riferimento all'ambito urbano e territoriale, al di là dell'enfasi sulla ricettività turistica alberghiera a cinque stelle. Ha prevalentemente portato a conclusione i restauri avviati dall'amministrazione precedente (tra cui lo spazio culturale di Montevergini, la Galleria d'Arte Moderna e l'arredo del prato del Foro Italico) ha continuato a insediare uffici comunali in molti immobili del centro storico e ha puntato sugli eventi artistici, scegliendo nuove location, come i ruderi spettacolari di palazzo Bonagia e le scuderie di palazzo Cefalà, in via Alloro. In quest'ultimo è stata aperta la galleria d'arte e architettura EXPA, gestita da giovani architetti che hanno riscosso la fiducia del Comune e che propongono ricchi calendari di eventi e di attività che però hanno scatenato le reazioni delle famiglie residenti per via della rumorosa attività indotta nelle ore serali e notturne.

Per la verità l'assessore al Centro storico alla fine del 2007 aveva avviato un'iniziativa per aggiornare la strumentazione urbanistica per il centro storico in rapporto all'ambito urbano e territoriale, predisponendo un bando per selezionare cinque esperti i cui nomi erano già filtrati sulla stampa, ma

30. Si vedano gli articoli di M. Romano, «Centro storico Ordinanza di sgombero per diciassette edifici», Gionnale di Sicilia, 29 dic. 2007 e S. Scarafia, "Quaranta famiglie nelle case cadenti), La Repubblica - Palermo, 11 apr. 2008. è stato fermato dal sind naio 2008 si è appreso l'assessorato al centro delegazione della socie resse a operare nel cent interventi di riqualifica denziale e alberghiera, mazione urbana ${ }^{32}$. Po elettorale e le elezioni della vita pubblica e de nulla. Solo l'assessore crolli, gli sgomberi e le

\section{Strumenti urbanis}

Nel 2007 è scadutc Regione nel 2002. A fr vazione in cui si chiede abusive per conoscere standard urbanistici, il dazione di piani partic borgata turistica di Mo e l'onerosità dell'opera

Per la verità la reda cabile variante genera serire la Valutazione piani urbanistici, ai se disposizione non può un regolamento adegu banistici in Sicilia è to

In ogni caso l'Amr un quadro generale di sguardo al centro stori di iniziative parziali ch

Una di queste rigu de centro direzionale

31. Si vedano gli articoli mo. 28 dic. $2007 \mathrm{e}$ «Scintille 32. L'operazione dovrebb Roberto Delli Santi, amico vo Ppe che fa gola agli en lermo, 6 gen. 2008. 
degli immobili a cui rintracciare e mobilifrazionata è un obietmune ha deciso di atare un percorso legale saranno ristrutturati in

Iunitari, il Comune ha a convinto la maggior e abitazioni. In difesa eso in campo il Comi2 ha organizzato mani.ull'uso degli immobili arte degli abitanti, con ratico il percorso verso ci con il problema abi-

icciato una politica orl'aggiornamento della -ealizzare una quota sitrebbe contribuire a rie calmierare il mercato llle funzioni da privileurbano e territoriale, al a cinque stelle. Ha preati dall'amministrazione rgini, la Galleria d'Arte ontinuato a insediare ufha puntato sugli eventi spettacolari di palazzo ro. In quest'ultimo è statita da giovani architetti pongono ricchi calendari zioni delle famiglie resiserali e notturne.

e del 2007 aveva avviaurbanistica per il centro predisponendo un bando filtrati sulla stampa, ma

Ordinanza di sgombero per difia, «Quaranta famiglie nelle è stato fermato dal sindaco, che ha bloccato tutto ${ }^{31}$. Nel primi giorni di gennaio $2008 \mathrm{si}$ è appreso, infatti, che il sindaco, nello stesso periodo in cui l'assessorato al centro storico si occupava del bando, avrebbe ricevuto una delegazione della società di Dubai Limit less che avrebbe manifestato interesse a operare nel centro storico e lungo la fascia costiera proponendo vari interventi di riqualificazione, recupero e nuova costruzione di edilizia residenziale e alberghiera, attraverso la costituzione di una società di trasformazione urbana ${ }^{32}$. Poco dopo, la caduta del governo Prodi, la campagna elettorale e le elezioni hanno polarizzato l'attenzione di tutti i protagonisti della vita pubblica e dei programmi per il centro storico non si è saputo più nulla. Solo l'assessore Scimemi è rimasto sulla breccia a fare i conti con $i$ crolli, gli sgomberi e le famiglie senza casa.

\section{Strumenti urbanistici semplici e complessi}

$\mathrm{Nel} 2007$ è scaduto anche il piano regolatore generale approvato dalla Regione nel 2002. A fronte delle prescrizioni del decreto regionale di approvazione in cui si chiedeva al Comune di ristudiare le lottizzazioni più o meno abusive per conoscere meglio la legittimita delle costruzioni e reperire gli standard urbanistici, il Comune ha ipotizzato di rinviare la questione alla redazione di piani particolareggiati, uno dei quali dovrebbe riguardare l'intera borgata turistica di Mondello. Prospettiva poco praticabile, per la complessità e l'onerosità dell'operazione, che comunque non sembra essere partita.

Per la verità la redazione di nuovi strumenti urbanistici, tra cui un'auspicabile variante generale del Prg si scontra al momento con l'obbligo di inserire la Valutazione ambientale strategica nei processi di formazione dei piani urbanistici, ai sensi del Dpr 4/2008 (Testo unico dell'Ambiente); tale disposizione non può essere applicata perché la Regione non si è dotata di un regolamento adeguato; pertanto la progettazione di nuovi strumenti urbanistici in Sicilia è totalmente bloccata.

In ogni caso l'Amministrazione comunale, invece di costruire comunque un quadro generale di previsioni urbanistiche in un'ottica di sistema, con uno sguardo al centro storico e al territorio metropolitano, ha intrapreso una serie di iniziative parziali che, tra l'altro, sono ben lungi dall'essere concluse.

Una di queste riguarda l'appalto concorso per la realizzazione del grande centro direzionale che dovrebbe ospitare tutti gli uffici regionali, così

31. Si vedano gli articoli di M. Lorello, «Uno staft riscriverà il Ppe», La Repubblica-Palermo. 28 dic. $2007 \mathrm{e}$ «Scintille sul Ppe, il sindaco frena», La Repubblica - Palermo, 29 dic. 2007.

32. L'operazione dovrebbe essere assistita dall'Unicredit e dal S. Paolo Imi e mediata dall'avv. Roberto Delli Santi, amico di Antonio Di Pietro. Si veda l'articolo di M. Lorello, «Dal nuovo Ppe che fa gola agli emiri parte la corsa solitaria di Cammaratan, La Repubhlica-Palermo, 6 gen. 2008 . 
come auspicato anche dal nuovo Presidente della Regione ${ }^{33}$. L'area in questione si trova all'interno di una delle zone più congestionate della città in cui si è realizzata un'espansione urbana compatta, con una concentrazione di edifici di notevole altezza (anche più di dieci piani), senza spazi pubblici, senza verde, senza parcheggi, inquinata e assordata dal traffico pesante che si svolge lungo la circonvallazione. La parte più a nord dell'area contiene alcuni manufatti storici di una certa rilevanza e presenta anche una fitta vegetazione con alberi di alto fusto. Con scelta poco felice (probabilmente imposta), il Prg destina l'area a uffici e ad attrezzature culturali.

L'appalto concorso non è stato aggiudicato sia perché si è presentata una sola ditta, sia perché il progetto proposto supera di gran lunga la volumetria consentita. Per andare avanti il Comune e la Regione hanno sottoscritto un protocollo nel quale il Comune si impegna a redigere una variante urbanistica con l'aumento dell'indice di fabbricabilità e la Regione si accolla l'onere finanziario per realizzare alcuni ponti pedonali sulla circonvallazione che faciliterebbero l'accessibilità dell'area e che il Comune ha commissionato con grande clamore a Dominique Perrault.

Un'altra iniziativa riguarderebbe la preparazione di un bando per un concorso di idee avente per oggetto la progettazione e l'utilizzazione dell'area gravitante sull'ex stazione ferroviaria Lolli. Si tratta di un'area centralissima compresa tra via Dante, via Parlatore e via Malaspina che ospita attualmente alcuni edifici storici delle Fs e il fascio ferroviario. Sulle aree attualmente occupate dai binari il Prg, probabilmente in seguito a forti pressioni, prevede le stesse infelici destinazioni di prima poco compatibili con la congestione del conteso urbano, fittamente edificato.

Le Ferrovie ( $R f i$ i) invece richiedono l'aumento dell'indice di fabbricabilità per un maggiore sfruttamento immobiliare dell'area e il Comune sembra voler assecondare tale istanza, anche se con modalità tutte da chiarire.

L'aggiornamento delle vicende palermitane non può ignorare l'evoluzione di una serie di iniziative avviate dal Comune mentre si redigeva il $\mathrm{Prg}$ (1997-99) che indussero Cervellati a rompere polemicamente con Orlando nel 1999. Si tratta per lo più di programmi e piani, di cui molti in variante al Prg, concepiti secondo le procedure dell'urbanistica negoziata. Tra questi sono compresi il Contratto di quartiere della Bandita (1998) e il Piano particolareggiato Costa-sud che sarebbero dovuti confluire nella variante generale. Ambedue le iniziative sono fallite prevalentemente per l'incapacità di risolvere il problema dei numerosi insediamenti abusivi esistenti sulle aree costiere demaniali.

Furono varati anche Programmi di riqualificazione urbana in alcuni quartieri popolari, un centinaio di Prusst e un Progetto integrato di inter-

33. Si tratta di una grande area di proprietà regionale, sita tra la circonvallazione, via Uditore, via Cimabue e via Leonardo da Vinci, ben nota alla cittadinanza di Palermo, perché nei pressi venne arrestato il capomafia Totò Riina. vento (Pit). In re hanno nulla di so: proposti per un $t$ centri commercia lizzazione di stra riqualificazione $\mathrm{u}$ lizzato negli anni rica di Bellolamp quartiere popolar

Gli interventi Regione sottoscri tra fondi pubblic battere condizion infrastrutture e ric

Nel caso dell vento (Pii), ment il Programma di venti pubblici su al S. Filippo Ner $€$. Gli interventi consistono in un una serie di inter un centro comm impianto sportiv altà gli stessi int attraverso un ipo dal patron del $\mathrm{Pa}$ comunale alla fi corso l'Inu Sicil l'Università di $\mathrm{P}$ gli ultimi framm andato avanti se dal parere dell' rilevato nella do no particolaregg progettuali $i^{35}$.

Sono passati re previste non gione aveva int la perdita del fi complicata per

34. Delibera del 35. Decreto asse 


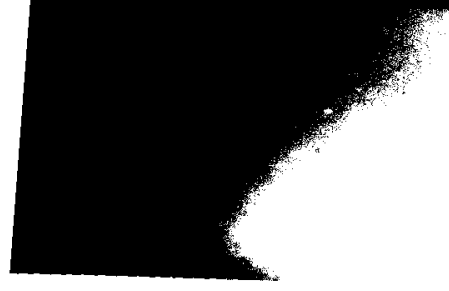

'gione ${ }^{33}$. L'area in quegestionate della città in zon una concentrazione i), senza spazi pubblici, dal traffico pesante che nord dell'area contiene senta anche una fitta veo felice (probabilmente ure culturali.

a perché si è presentata ra di gran lunga la volula Regione hanno sottona a redigere una varianbilità e la Regione si acpedonali sulla circonvalea e che il Comune ha 'errault.

one di un bando per un itazione e l'utilizzazione Lolli. Si tratta di un'area re e via Malaspina che oil fascio ferroviario. Sulle bilmente in seguito a fortj di prima poco compatibili edificato.

dell'indice di fabbricabiell'area e il Comune semodalità tutte da chiarire. on può ignorare l'evolue mentre si redigeva il Prg lemicamente con Orlando i, di cui molti in variante stica negoziata. Tra questi andita (1998) e il Piano i confluire nella variante alentemente per l'incapanenti abusivi esistenti sul-

azione urbana in alcuni ogetto integrato di inter-

a la circonvallazione, via Udidinanza di Palermo, perché nei vento (Pit). In realtà gli interventi compresi nel Prusst palermitano non hanno nulla di sostenibile: si tratta di progetti per circa 600 miliardi di lire, proposti per un terzo da privati per la realizzazione di alberghi, cliniche, centri commerciali, parcheggi, ecc. e per due terzi dal Comune per la realizzazione di strade, svincoli, altri parcheggi. Attualmente i Programmi di riqualificazione urbana investono i quartieri popolari di Borgo Nuovo, realizzato negli anni ' 60 nella periferia occidentale di Palermo, sotto la discarica di Bellolampo, il più famoso Zen, ora denominato San Filippo Neri e il quartiere popolare dello Sperone, lungo la costa sud-orientale.

Gli interventi si basano su un Accordo di programma tra il Comune e la Regione sottoscritto nel 2005 , che prevede la spesa di 76 milioni di euro, tra fondi pubblici ex Gescal (47,4 milioni) e privati (28,3 milioni) per abbattere condizioni di degrado, realizzando spazi verdi, servizi, parcheggi, infrastrutture e riqualificando edifici residenziali e attrezzature scolastiche.

Nel caso dell'ex Zen siamo in presenza di un Piano integrato di intervento (Pii), mentre negli altri due casi lo strumento urbanistico adoperato è il Programma di recupero urbano (Pru). In dettaglio sono previsti 33 interventi pubblici su Borgo Nuovo per 6,1 milioni di $€ ; 22$ interventi pubblici al S. Filippo Neri per 24,7 milioni di $€ ; 27$ sullo Sperone per 6,1 milioni di $€$. Gli interventi promossi dai privati e inseriti nell' Accordo di programma consistono in un centro sportivo a Borgo Nuovo per circa un milione di $€ \mathrm{e}$ una serie di interventi al S. Filippo Neri per 27,3 milioni di $€$, comprendenti un centro commerciale, parcheggi, un centro per anziani, un albergo, un impianto sportivo e altri interventi di edilizia sanitaria e residenziale. In realtà gli stessi interventi previsti per il S. Filippo Neri sono stati riproposti attraverso un ipotetico piano particolareggiato in variante al Prg, promosso dal patron del Palermo Calcio, Maurizio Zamparini, adottato dal Consiglio comunale alla fine del $2006^{34}$. Contro questa variante hanno presentato ricorso l'Inu Sicilia, Legambiente e il Dipartimento Città e Territorio dell'Università di Palermo, in quanto si trattava della devastazione di uno degli ultimi frammenti del verde storico della Piana dei Colli, ma il Comune è andato avanti senza alcun ripensamento. L'iniziativa è stata però rallentata dal parere dell'Assessorato regionale al Territorio e Ambiente che non ha rilevato nella documentazione presentata i contenuti e gli elaborati del piano particolareggiato e ha richiesto una cospicua integrazione degli elaborati progettuali ${ }^{35}$.

Sono passati tre anni dalla data dell'Accordo di programma e delle opere previste non si vede ancora quasi nulla. Tra l'altro il Presidente della Regione aveva intimato al Comune di avviare i lavori entro marzo 2008, pena la perdita del finanziamento pubblico. La questione si è però ulteriormente complicata per via della pubblicazione del nuovo prezziario regionale e di

34. Delibera del Cc. n. 365 del 6 dic. 2006.

35. Decreto assessoriale del 6 dic. 2007 
una recente sentenza della Corte Costituzionale che ha stabilito che gli espropri devono essere pagati a prezzi di mercato. Quest'ultima disposizione imporrà una notevole riduzione delle previsioni progettuali e i quartieri popolari potrebbero veder svanire nel nulla giardini, scuole e campi sportivi ${ }^{36}$.

Passiamo ora ad esaminare lo stato di attuazione del Prusst, avviato nel 1999. La prima stranezza è che il Prusst di Palermo interessa l'intero turritorio comunale; si è pervenuti a tale scelta per non escludere quei privati che avessero interessi localizzati al di fuori di ambiti circoscritti a priori dal Comune. Si comincia, quindi, con un bel segnale di disponibilità nei confronti dell'iniziativa privata comunque e dovunque. Pertanto fanno parte del Prusst interventi disparati ed eterogenei, ricadenti in parte in aree urbanizzate, in parte in aree agricole periferiche. Tra le proposte più stravaganti quella della Curia di Palermo che ha previsto la realizzazione della nuova sede della Facoltà di Teologia con annesso campus universitario in un'area periferica del tutto inadeguata, ricadente in pieno verde agricolo.

II Prusst si articola in sette macrointerventi, ciascuno dei quali comprende interventi pubblici e privati ${ }^{37}$. In ciascun ambito il rapporto in percentuale tra interventi pubblici e privati è diverso, con una percentuale più alta di intervento pubblico negli ambiti meno urbanizzati dove è quindi necessario costruire le condizioni per promuovere gli investimenti privati. È invece maggiore la percentuale di intervento privato la dove è migliore la qualità urbana e infrastrutturale.

Il lungo tempo trascorso tra la emanazione del bando, la formazione e l'approvazione del programma ha costituito un grave handicap per gli investitori privati per i quali il fattore tempo è di grande importanza ${ }^{38}$. Alcuni privati hanno aperto contenziosi con il Comune per via dei ritardi accumulati nell'approvazione dei progetti; altri hanno preferito abbandonare la via del Prusst e investire altrove. A scoraggiare ulteriormente gli investitori privati ha influito la decisione dell'Amministrazione comunale di richiedere un ulteriore contributo straordinario da corrispondere in relazione all'entità e alla natura degli interventi proposti in variante ${ }^{39}$.

A distanza di alcuni anni si cominciano a vedere completati solo una decina dei 64 interventi pubblici inseriti nel Prusst di cui, alcuni, erano già in

36. Si veda D. Prestigiacomo, «Il flop delle grandi opere nelle periferie», La Repubblica Palermo, 5 apr. 2008.

37. I macrointerventi risultano cosi articolati: macrointervento 1 "ll nuovo sistema urbano della circonvallazione"; macrointervento 2 "Il recupero dell"edificato storico per la residenza"; macrointervento 3 "Il sistema turistico culturale nel centro storico"; macrointervento 4 "Il potenziamento della maglia infrastrutturale nella città settentrionale"; macrointervento 5 "La tiqualificazione della costa sud"; macrointervento 6 "L'asse centrale Ducrot-Sampolo"; macrointervento 7 "Il sistema di accessibilità al porto da sud-est"

38. Il bando era allegato al Dm. n. 1169 del 1998 «Promozione di programmi in ambito urbano denominati Programmi di riqualificazione urbana e di sviluppo sostenibile del territorio».

39. Delibera di Gm. n. 480/2002. fase di realizzazione del restauro del comp moderna, finalmente

Per quanto riguard la situazione è abbast: finanziamento, 14 so rimanenti sono stati a rinuncia di 14 privati vato prescritto dal ba Ministero circa 7 mili

Per ovviare a tal possibilmente affidab avviso pubblico ${ }^{4()} \mathrm{ch}$ devono possedere e a imprenditori improv programma i soggetti di realizzazione alla Tale iniziativa è in ev

In attesa di ulteric a causa dei tempi in contesto, con l'aggra imprenditoriale, com

Un altro strument risorse finanziarie pe riale (Pit) che comp produttive, nonché $p$ aree. Tra questi, var zione del Parco arc grande area a verd d'Orlèans (oltre $25 \mathrm{r}$ Palazzo Chiaramont

Il nuovo verde $\mathrm{pt}$ verde urbano dopo l'impianto di $40 \mathrm{mil}$ li; il costo previsto è nel Pit con finanzia dati all'associazione tania, Agricoltura e cantiere è stato ape dalla Prefettura di ( mafia di una delle

40. Delibera di Gm. I 41. Avviso pubblico: 
to che gli edisposizione uartieri poposportivi ${ }^{36}$.

;t, avviato nel I'intero turrie quei privati itti a priori dal bilità nei conto fanno parte e in aree urbapiù stravaganti me della nuova tario in un'area solo.

quali comprenporto in percencentuale più alta dove è quindi estimenti privati. ove è migliore la

, la formazione $\mathrm{e}$ licap per gli inveoortanza ${ }^{38}$. Alcuni ei ritardi accumubbandonare la via nte gli investitori unale di richiedeere in relazione .

etati solo una decuni, erano già in

ie», La Repubblica uovo sistema urbano torico per la residen"; macrointervento 4 "; macrointervento 5 le Ducrot-Sampolo";

nmi in ambito urbano le del territorio». fase di realizzazione tramite altri canali finanziari. $\grave{E}$ il caso, per esempio, del restauro del complesso conventuale di S. Anna adibito a Galleria d'Arte moderna, finalmente ultimato dopo più di vent'anni dal progetto iniziale.

Per quanto riguarda invece lo stato di attuazione degli interventi privati, la situazione è abbastanza sconfortante. Su 77 interventi privati ammessi al finanziamento, 14 sono stati ritirati dai proponenti; meno della metà dei rimanenti sono stati approvati, ma non c'è ancora nessuna realizzazione. La rinuncia di 14 privati ha messo in crisi il rapporto percentuale pubblico/privato prescritto dal bando con 11 rischio per il Comune di dover restituire al Ministero circa 7 miliardi di vecchie Lit.

Per ovviare a tale inconveniente e reperire nuove iniziative private, possibilmente affidabili, il Comune è stato costretto a predisporre un nuovo avviso pubblico ${ }^{40}$ che introduce una serie di requisiti che gli imprenditori devono possedere e alcune condizioni per scoraggiare eventuali proposte di imprenditori improvvisati, come ad esempio che «possono partecipare al programma $i$ soggetti che intendono attuare l'intervento proposto, dalla fase di realizzazione alla fase di gestione per un periodo di almeno dieci annis ${ }^{43}$. Tale iniziativa è in evoluzione e non ha ancora prodotto risultati notevoli.

In attesa di ulteriori sviluppi non c'è da essere molto ottimisti sugli esiti a causa dei tempi incerti delle procedure, delle difficoltà economiche del contesto, con l'aggravante del condizionamento mafioso su ogni iniziativa imprenditoriale, come testimoniano le cronache quotidiane.

Un altro strumento sul tappeto a cui sono ancorati progetti, programmi e risorse finanziarie per 11 milioni e 400 mila $€$ è il Progetto integrato territoriale (Pit) che comprende varie forme di sostegno economico alle attività produttive, nonché progetti di infrastrutture e di riqualificazione di ampie aree. Tra questi, varie sistemazioni a parcheggi, interventi per la realizzazione del Parco archeologico del Castellammare, la sistemazione di una grande area a verde all'interno dell'insediamento universitario di Parco d'Orlèans (oltre 25 ha) e il completamento del restauro del comprensorio di Palazzo Chiaramonte-Steri, sede del Rettorato dell'Università.

Il nuovo verde pubblico di Parco d'Orlèans sarebbe la più grande area di verde urbano dopo il parco storico della Favorita. II progetto prevede l'impianto di 40 mila piante, boschi, prati, percorsi pedonali e piste ciclabili; il costo previsto è di 8 milioni di $€$. Come già detto l'intervento è inserito nel Pit con finanziamenti ex Agensud e Por Sicilia. I lavori sono stati affidati all'associazione temporanea di imprese formata da Technital spa di Catania, Agricoltura e giardinaggio di Palermo e lcaro Ecology srl di Gela e il cantiere è stato aperto nel corso del 2005; i lavori però sono stati fermati dalla Prefettura di Catania che ha rilevato la mancanza del certificato antimafia di una delle imprese e sono ripresi dopo un anno e mezzo di fermo,

40. Delibera di Gm. n. 31 del 15 feb. 2006.

41. Avviso pubblico allegato alla delibera di Gm. n. 31/2006. 
alla fine di aprile 2008. Questa circostanza ha comportato la risoluzione del contratto con le ditte e la necessità di una transazione per riavviare $i$ lavori quando la Prefettura ha fatto sapere che gli ostacoli erano stati rimossi. Le conseguenze di questo intoppo sono state la dilatazione dei tempi di ultimazione dei lavori, previsti forse troppo ottimisticamente per la primavera 2009 e l'aumento del costo dell'opera lievitato a 11 milioni e 400 mila $€$. Questo è l'ennesimo esempio di come la mafia funesti lo svolgimento di qualunque attività. Anche i cantieri per il restauro di palazzo Chiaramonte $\mathrm{e}$ di altri edifici universitari hanno avuto a che fare direttamente con emissari delle famiglie mafiose che pretendevano di riscuotere il "pizzo" come ha denunciato pubblicamente il Rettore Giuseppe Silvestri.

\section{Mobilità e parcheggi}

Palermo è una città avvelenata dal traffico e sostanzialmente priva di un efficace sistema di trasporto pubblico. L'inquinamento, provocato dai gas di scarico dei veicoli, raggiunge picchi elevatissimi e si discute da anni di pedonalizzare alcune aree centrali. La questione ha sempre sollevato molte polemiche anche se il fronte dei commercianti, che è stato sempre quello più contrario a qualunque ipotesi di pedonalizzazione del centro cittadino, comincia a incrinarsi.

Attualmente il servizio di trasporto pubblico si basa su pochi autobus malconci e inquinanti che riescono a essere efficaci solo lungo la direzione nord-sud. La città è anche attraversata da imponenti flussi di pullman che assicurano i collegamenti territoriali in tutte le direzioni, con relative caotiche zone di stazionamento e dal traffico pesante dei Tir per il trasporto merci. Alla fine del 2001 è entrato in funzione il collegamento ferroviario tra Palermo e l'aeroporto di Punta Raisi, dopo una quindicina d'anni di lavori e con un numero di corse a cadenza di mezz'ora. Questo collegamento è comunque utilizzato per gli spostamenti urbani da coloro che abitano in prossimità delle fermate.

La soluzione razionale e lungimirante della mobilità è uno dei temi strategici per il funzionamento delle strutture urbane. Purtroppo questa tematica continua ad essere gestita in maniera non adeguata, dimostrando poca consapevolezza del ruolo fondamentale che un sistema di trasporto pubblico efficiente può svolgere nella riqualificazione e nella riorganizzazione delle funzioni urbane, nell'abbattimento dell'inquinamento e nell'innalzamento della qualità della vita individuale e associata.

Il tema della mobilità è stato affrontato dall'Amministrazione comunale attraverso il Piano integrato del trasporto pubblico di massa, approvato dal Consiglio comunale nel maggio 2002. Esso prevedeva la realizzazione di tre linee tranviarie che servono essenzialmente l'estrema periferia sud- orientale (Roccella, Br: Nuovo e del Cep. Prev Stazione Centrale all'a ghezza del tracciato a dell'anello ferroviario una al Porto. A lungo t tropolitana leggera aut rezione nord-sud, da $v$ rà a un miliardo e $85 \mathrm{~m}$

Tralasciando quest' mandoci sui progetti rilevare che il progetto ra è successo conferm tesi sembra che le reti nella dovuta consider secondo una vecchia $n$ qualità e alle condizio sclusivamente all'ecor

Il progetto del Pass Fs tra i progetti priorit Programma Quadro st tra gli interventi previ so prevede un traccia nee; consentirà una po per raggiungere l'a

$\mathrm{Nel} 2004$ la Italfer doppio del Passante; appalto è stato aggiu sta da Consorzio stab la capofila, una delle Sacyr. I lavori vengo progetto, finalmente stazioni. Suscita part tratto del tracciato ne con l'assetto urbano che di scavo per l'in della città, basate su zioni in superficie. $\mathrm{F}$ comitato di cittadin Sindaco di far modi della talpe meccanic renze con i manufatı resistenza ma poi ha 
risoluzione del avviare i lavori tati rimossi. Le i tempi di ultier la primavera ii e 400 mila $€$. svolgimento di ) Chiaramonte e nte con emissari pizzo" come ha

nente priva di un rovocato dai gas iscute da anni di e sollevato molte to sempre quello centro cittadino,

su pochi autobus iungo la direzione si di pullman che con relative caotiar il trasporto mernto ferroviario tra d'anni di lavori e ollegamento è coe abitano in pros-

uno dei temi strapo questa tematidimostrando poca trasporto pubbliriorganizzazione to e nell'innalza-

razione comunale sa, approvato dal realizzazione di na periferia sud- orientale (Roccella, Brancaccio) e i quartieri popolari occidentali di Borgo Nuovo e del Cep. Prevedeva anche il raddoppio del binario che collega la Stazione Centrale all'aeroporto (Passante ferroviario) aumentando la lunghezza del tracciato a sud fino a Brancaccio; prevedeva il completamento dell anello ferroviario esistente con l'inserimento di nuove fermate di cui una al Porto. A lungo termine si prevede inoltre la realizzazione di una metropolitana leggera automatica che dovrebbe attraversare tutta la citta in direzione nord-sud, da via Oreto a Mondello; il costo di tale opera ammonterà a un miliardo e 85 milioni di $€$.

Tralasciando quest'ultima previsione ancora di larga massima e soffermandoci sui progetti più definiti, come il Passante ferroviario, dobbiamo rilevare che if progetto ipotizzato non è risultato convincente e quanto finora è successo conferma la scarsa qualità della progettazione. In estrema sintesi sembra che le reti e i nodi ferroviari siano stati progettati senza tenere nella dovuta considerazione l'integrazione con le zone urbane sovrastanti, secondo una vecchia modalità di progettazione dei trasporti indifferente alla qualità e alle condizioni delle zone urbane da servire, se non interessata esclusivamente all'economicità dell'operazione.

Il progetto del Passante, di cui si discute dagli anni '70, viene inserito da Fs tra i progetti prioritari del Nodo di Palermo, nell'ambito dell'Accordo di Programma Quadro sul trasporto ferroviario, sottoscritto nel 2001 e si trova tra gli interventi previsti dalla Legge obiettivo del Governo Berlusconi. Esso prevede un tracciato di $26 \mathrm{~km}$ con 18 fermate, dieci delle quali sotterranee; consentirà una cadenza dei treni ogni 10 minuti e di dimezzare il tempo per raggiungere l'aeroporto.

$\mathrm{Nel} 2004$ la Italferr spa, per conto di Rfi ha appaltato i lavori per il raddoppio del Passante; l'importo complessivo è 623 milioni di euro. 11 maxi appalto è stato aggiudicato all'associazione temporanea di imprese composta da Consorzio stabile Sis, Sintagma spa (Perugia) e Geodata srl (Torino); la capofila, una delle tre ditte riunite nella sigla Sis, è il colosso madrileno Sacyr. I lavori vengono consegnati nel gennaio 2005. Le caratteristiche del progetto, finalmente rese note alla cittadinanza, destano perplessità e contestazioni. Suscita particolare allarme la previsione di non interrare un lungo tratto del tracciato nella periferia nord della città, con conseguenti conflitti con l'assetto urbano esistente in superficie; preoccupano le modalità tecniche di scavo per l'interramento della linea in alcune zone molto popolose della città, basate su invadenti lavorazioni in trincea e conseguenti demolizioni in superficie. Per contrastare tali previsioni si è formato nel 2005 un comitato di cittadini, assistito da tecnici, che ha chiesto formalmente al Sindaco di far modificare a Rfi il progetto, prevedendo per esempio l'uso della talpe meccaniche per gli scavi, che non avrebbero provocato interferenze con i manufatti e le attività in superficie. Il Sindaco ha fatto un po" di resistenza ma poi ha riconosciuto la fondatezza delle ragioni del comitato, 
quando già alcuni cantieri stavano per essere aperti. La variante viene formalizzata in un nuovo Protocollo di intesa tra il Comune, il Ministero delle Infrastrutture, la Regione e Rfi nel dicembre 2006 e richiede un 'integrazione finanziaria pari a 125 milioni di $€$, che vengono reperiti attraverso l'inclusione dell'opera tra i grandi progetti del Por 2007-2013. A parte la dilatazione dei tempi e della spesa, la progettazione del Passante dimostra la propria antiquata autoreferenzialità, come infrastruttura a sé stante, poco relazionata alla città in superficie e al ruolo che potrebbe svolgere nella riqualificazione e nella rifunzionalizzazione dell'insediamento urbano, come accade in molte citta europee e perfino italiane. Su questa vicenda, particolarmente succulenta dal punto di vista economico non poteva mancare l'interessamento delle famiglie mafiose.

Sull'appalto del Passante, la Procura della Repubblica ha aperto una inchiesta dopo aver trovato i "pizzini" inviati dai boss di Palermo nord, Salvatore e Sandro Lo Piccolo, a Bernardo Provenzano con il seguente messaggio: «Ci dica se ha qualche impresa per il calcestruzzo. Argomento metropolitana». I Lo Piccolo, padre e figlio, cercavano di concordare una strategia comune con il vertice mafioso, per coordinare al meglio la spartizione dei nuovi cantieri. Come sempre le famiglie mafiose aspirano a impadronirsi delle opere per il movimento terra, i trasporti, i subappalti e le guardianie ${ }^{42}$.

E documentato che la mafia ha cercato di condizionare anche i lavori per il raddoppio del Ponte di Corleone, sulla circonvallazione, i cui lavori, per un importo di 14 milioni di $€$, erano iniziati nell'autunno 2005. L'opera si trova al confine tra il mandamento mafioso di Pagliarelli e quello di Villagrazia e questo ha provocato interferenze e perfino tentativi di fare modificare il progetto ${ }^{43}$.

Per concludere questa ricognizione sulle infrastrutture di trasporto bisogna fare un cenno sulla realizzazione dei parcheggi. Sono stati realizzati o sono in costruzione un gran numero di aree di parcheggio, di cui alcune per migliaia di posti-macchina. La formula adoperata in molti casi è quella del project financing. Il parcheggio di dimensioni maggiori già realizzato si trova in via Basile ed è tangente al campus universitario di Parco d'Orlèans (1.072 posti macchina). Peccato che non sia assicurata nessuna forma di intermodalità per lasciare la macchina e raggiungere il centro urbano con un mezzo pubblico.

Recentemente l'amministrazione comunale ha ripreso l'argomento della mobilità inserendo il tema del trasporto pubblico all'interno di un pro-

42. La vicenda è stata ulteriormente confermata dalle confessioni di un nuovo pentito, Loreto Rizzo, piccolo imprenditore nel settore dei trasporti, genero di Salvatore Geraci, importante capo mafia, nuovo ministro dei Lavori pubblici di Cosa Nostra, ucciso il 5 ott. 2004, perché si era «allargato» troppo. Geraci aveva preso il posto di Angelo Siino, da tempo in galera.

43. Si veda l'articolo di A. Ziniti, «Mediazione sul ponte Corleone», La Repubblica-Palermo, 8 feb. 2008. gramma più ampio: il $P$ le, che è stato finanziat gruppamento temporan tagma di Perugia, già in vori del Passante ${ }^{44}$.

Dalla relazione alleg la definizione di un qua tarie nel breve, medio e mobilità sostenibile [.. incidere in modo strutt cittadini e operatori. Si alle tre sfere ambientale pone un approccio integ zioni da mettere in cam stema dei trasporti $[\ldots]$ sviluppo per fasi e la v iniziativa appena avviat palermitani reclutati nel trasporti, senza conside da esperti nella pianifica

$\dot{\mathrm{E}}$ un vero peccato $c$ portunità legate a una vrebbe interagire siner plessiva riguardante il

\section{Il waterfront}

Negli ultimi anni se città di mare approfitta tuale del tema del wat da esaltare.

Nel 2004 l'Autorità la sistemazione di part to) e il concorso viene bra che da tale iniziativ

Tra la fine del 2006 alcune mostre della Bis ma il rapporto tra le citt

44. Il piano è stato tinan contesto dell'Accordo di $\mathrm{Pr}$ la qualità della vitan. Cfr. i relazione allegata al piano ( 
La variante viene forrune, il Ministero delle richiede un' integrazioono reperiti attraverso 2007-2013. A parte la : del Passante dimostra uttura a sé stante, poco rebbe svolgere nella ridiamento urbano, come questa vicenda, particoo non poteva mancare

Ibblica ha aperto una inss di Palermo nord, Salno con il seguente messstruzzo. Argomento medi concordare una strateI meglio la spartizione dei pirano a impadronirsi delpalti e le guardianie $e^{42}$. ndizionare anche $i$ lavori onvallazione, i cui lavori, ell'autunno 2005. L'opera Pagliarelli e quello di Vilino tentativi di fare modi-

strutture di trasporto bisogi. Sono stati realizzati o cheggio, di cui alcune per in molti casi è quella del naggiori già realizzato si -sitario di Parco d'Orlèans urata nessuna forma di ine il centro urbano con un

ripreso l'argomento della co all'interno di un pro-

essioni di un nuovo pentito, Lonero di Salvatore Geraci, imporNostra, ucciso il 5 ott. 2004, perigelo Sino, da tempo in galera. Corleone», La Repubblica - Pa- gramma più ampio: il Piano degli interventi strategici di mobilità sostenibi$l e$, che è stato finanziato dalla Regione, messo a bando e vinto da un raggruppamento temporaneo di imprese di cui è capogruppo la società Sintagma di Perugia, già incontrata tra le imprese che si erano aggiudicate i lavori del Passante ${ }^{44^{\circ}}$.

Dalla relazione allegata al piano si apprende che «Obiettivo del Piano è la definizione di un quadro organico di interventi, politiche e azioni prioritarie nel breve, medio e lungo periodo, finalizzato a realizzare un sistema di mobilità sostenibile $[. .$.$] in grado di contrastare l'emergenza ambientale e$ incidere in modo strutturale e duraturo sugli attuali modelli di mobilità di cittadini e operatori. Si precisa anche che la sostenibilità va intesa rispetto alle tre sfere ambientale, sociale ed economica dello sviluppo. II piano propone un approccio integrato ai problemi della mobilità e alle possibili soluzioni da mettere in campo per minimizzare i diversi impatti generati dal sistema dei trasporti [...]. Esso si fonda su due criteri progettuali di base, to sviluppo per fasi e la visione di area vasta». Con riferimento a quest'ultima iniziativa appena avviata, la cosa che colpisce negativamente è che gli esperti palermitani reclutati nel gruppo di lavoro sono stati scelti tutti nel settore dei trasporti, senza considerare minimamente la necessità di apporti provenienti da esperti nella pianificazione territoriale e nella progettazione urbanistica.

$\grave{\text { E }}$ un vero peccato che a Palermo non si riescano a cogliere tutte le opportunità legate a una visione europea del sistema della mobilità che dovrebbe interagire sinergicamente con una progettualità urbanistica complessiva riguardante il sistema insediativo e le funzioni urbane.

\section{11 waterfront}

Negli ultimi anni sembra che Palermo abbia deciso di riproporsi come città di mare approfittando del successo mediatico, pubblicistico e progettuale del tema del waterfront, considerato giustamente un valore aggiunto da esaltare.

$\mathrm{Nel} 2004$ l'Autorità portuale bandisce un concorso di progettazione per la sistemazione di parte del waterfront cittadino (zone della Cala e del Porto) e il concorso viene vinto da alcuni giovani professionisti, ma non sembra che da tale iniziativa siano scaturiti esiti operativi.

Tra la fine del 2006 e l'inizio del 2007 Palermo ospita per la prima volta alcune mostre della Biennale di Architettura di Venezia che hanno come tema il rapporto tra le citta e i waterfront e anche Palermo, tramite la cosiddetta

44. Il piano è stato finanziato dalla Regione Sicilia (Dipartimento Programmazione) nel contesto dell’ Accordo di Programma Quadro «Riqualificazione urbana e miglioramento de!la qualità della vita». Cfr. il sito web del Comune di Palermo, dove è possibile scaricare la relazione allegata al piano (lug. 2007). 
Officina del Porto, espone una proposta di riassetto della fascia costicra (La Biennale di Venezia, 2006).

Le ipotesi progettuali prevedono una serie di interventi che cominciano dalla borgata costiera dell' Arenella, trovano la massima concentrazione sul Molo trapezoidale e si concludono con la proposta del porto turistico di S. Erasmo e la riqualificazione della foce dell'Oreto. La finalità generale è quella di valorizzare il ruolo e l'immagine di Palermo come città di mare, abbattendo le barriere portuali, proponendo nuove attività e funzioni, attraverso visioni seducenti di darsene, laboratori artistici e musicali, solarium, ecc.

Le proposte sono enunciate con enfasi visionaria e sono rappresentate con un allestimento molto suggestivo, ma una delle finalità chiaramente espressa è lo sfruttamento immobiliare del Molo trapezoidale a stretto contatto con il Parco archeologico del Castellammare.

Inoltre non risulta convincente la previsione di scaricare tutto il traffico pesante sulla costa (via Crispi, Cala, Foro Italico) e poi sulla Ss 113 , sempre a raso. La specializzazione dei flussi e la loro articolazione su quote diverse, con il traffico pesante interrato, è l'unica soluzione che consentirebbe di ricostituire il rapporto tra la città e il mare. Questa soluzione è stata adottata con successo a Barcellona, città che ha problematiche simili a quelle di Palermo, anche dal punto di vista insediativo, topografico e idrografico (Cannarozzo, 2007). Li però è stato varato e attuato un progetto a medio e lungo termine, ampiamente condiviso dagli enti e dalle istituzioni interessate, mettendo virtuosamente a frutto i meccanismi della concertazione.

La preoccupazione attuale è che gli attuali lavori di collocazione dei collettori fognari verso la stazione di pompaggio, sita di fronte a Porta Felice, che dovrebbero confluire nel depuratore di Acqua dei Corsari, abbiano pregiudicato per sempre l'ipotesi risolutiva dell'interramento del traffico pesante.

Per quanto riguarda l'utilizzazione del Molo trapezoidale, la rigida specializzazione in zone, archeologica ed edificabile con un invadente disegno urbano a scacchiera, che alterna isolatoni quadrangolari e rigide darsene, è molto deludente ed esplicitamente motivata da obiettivi di redditività immobiliare. Sarebbe stato preferibile includere tutto il Molo Trapezoidale in un progetto architettonico di maggiore respiro e unitarietà con caratteristiche di grande parco archeologico (ma non solo), integrato da sistemazioni verde, attrezzature pubbliche e parcheggi anche a servizio del traffico turistico indotto dalle navi di crociera.

Ulteriori rilevanti novità provengono dall'Autorità Portuale di Palermo presieduta attualmente dal dinamico e intraprendente ingegnere Nino Bevilacqua: la prima riguarda l'ampliamento della circoscrizione territoriale dell'Autorità Portuale di Palermo con l'inclusione del porto di Termini Imprese (estate 2007) al fine di realizzare un unico sistema portuale significativo. Per dirla con le parole del Presidente $1 /$ movo sistema portuale non solo amplierà l'offerta di aree di movimentazione, approdi e servizi, ma in- crementerà l'offert logistica della Sicil zioso percorso è st nuovo piano regola l'altro, la rimozione banchine, il restylir zi nell'area portual da crociera ${ }^{45}$.

\section{Conclusioni}

Palermo dovreb Fino a quando il $m$ pezzi. fino a quand fino a quando le $\mathrm{p}$ una città europea.

Riferimenti bibliog

La Biennale di Vene

T. Cannarozzo (1994 rare, n. 1.

T. Cannarozzo (199 del centro storico

T. Cannarozzo (2000 studi urbani e reg

T. Cannarozzo (2004 urbani e regional

T. Cannarozzo (200 formazioni, n. 19

T. Cannarozzo (200 gelini (a cura di) ii, Atti del Conv 27-28 ott. 2005,

Commissione Nazio il 5 aprile 1993 ,

M. Orlando (2006), di Palermo", $U r$

45. Si veda l'insert 
fascia costiera ( $\mathrm{La}$

Iti che cominciano concentrazione sul ,orto turistico di S. finalità generale è xe città di mare, abfunzioni, attraverso , solarium, ecc. sono rappresentate alità chiaramente esidale a stretto con-

icare tutto il traffico i sulla Ss 113 , semlazione su quote dione che consentirebsta soluzione è stata natiche simili a quelyrafico e idrografico n progetto a medio e istituzioni interessate, certazione.

collocazione dei colfronte a Porta Felice, Corsari, abbiano preo del traffico pesante. zoidale, la rigida speun invadente disegno ari e rigide darsene, è tivi di redditjvità imMolo Trapezoidale in arietà con caratteristigrato da sistemazioni vizio del traffico turi-

Portuale di Palermo ingegnere Nino Bevioscrizione territoriale porto di Termini Imna portuale significasistema portuale non orodi e servizi, ma in- crementerà l'offerta di filiera, di vera e propria piattaforma di trasporto e logistica della Sicilia occidentale. Uno dei primi segmenti di questo ambizioso percorso è stata la predisposizione dello schema di massima di un nuovo piano regolatore del porto di Palermo che dovrebbe comportare, tra l'altro, la rimozione delle barriere tra la citta e il porto, l'ampliamento delle banchine, il restyling della Stazione Marittima e il miglioramento dei servizi nell'area portuale anche in relazione all'aumento del traffico delle navi da crociera ${ }^{45}$.

\section{Conclusioni}

Palermo dovrebbe liberarsi dalla mafia e diventare una città europea. Fino a quando il mare sarà inquinato, fino a quando il centro storico cadrà a pezzi, fino a quando non ci sarà un sistema di trasporto pubblico efficiente, fino a quando le periferie saranno sinonimo di degrado, Palermo non sarà una città europea.

\section{Riferimenti bibliografici}

La Biennale di Venezia (2006), Città-Porto City Port, Marsilio, Padova.

T. Cannarozzo (1994), «ll piano dei musei nel centro storico di Palermo», Recuperare, n. 1.

T. Cannarozzo (1996) Palermo tra memoria e futuro. Riqualificazione e recupero del centro storico, Publisicula, Palermo.

T. Cannarozzo (2000), «Palermo: le trasformazioni di mezzo secolo», Archivio di studi urbani e regionali, n. 67.

T. Cannarozzo (2004a), «Palemo: il martirio di un piano orfano», Archivio di studi urbani e regionali, n. 80.

T. Cannarozzo (2004b), «Centro storico di Palermo: dopo il PPE», Urhanistica Informazioni, n. 193.

T. Cannarozzo (2007), «Palermo-Barcellona: cosi simili, cosi distanti», in A. Angelini (a cura di), Mediterraneo - Città, culture, ambiente, governance, migranti, Atti del Convegno Internazionale Le Metropoli del Mediterraneo, Palermo, 27-28 ott. 2005, FrancoAngeli, Milano.

Commissione Nazionale Antimafia dell'XI legislatura (1993) Relazione approvata il 5 aprile 1993, pubb. nel supplemento di Repubblica del 10 apr.

M. Orlando (2006), «La periferia in centro. Le politiche attuali per il centro storico di Palermon, Urbanistica Informazioni, n. 208.

45. Si veda l'inserto speciale «Porto di Palermo», Giornale di Sicilia, 23 apr. 2008. 